'Institute for Immunity, Transplantation and Infection, Stanford University School of Medicine, Stanford University, Stanford, CA, USA.

${ }^{2}$ Department of Pathology, Stanford University School of Medicine, Stanford University, Stanford, CA, USA.

${ }^{3}$ Department of Microbiology \& Immunology, Stanford

University School of Medicine, Stanford University, Stanford, CA, USA.

${ }^{4}$ Chemistry, Engineering \& Medicine for Human Health, Stanford University School of Medicine, Stanford University, Stanford, CA, USA.

${ }^{5}$ CSK Vaccines, Rockville, MD, USA.

凶e-mail:bpulend@

stanford.edu

https://doi.org/10.1038

s41573-021-00163-y

\title{
Emerging concepts in the science of vaccine adjuvants
}

\author{
Bali Pulendran (10 1,2,3,4凶, Prabhu S. Arunachalam (1) ${ }^{1}$ and Derek T. O’Hagan ${ }^{5}$
}

Abstract | Adjuvants are vaccine components that enhance the magnitude, breadth and durability of the immune response. Following its introduction in the 1920s, alum remained the only adjuvant licensed for human use for the next 70 years. Since the 1990s, a further five adjuvants have been included in licensed vaccines, but the molecular mechanisms by which these adjuvants work remain only partially understood. However, a revolution in our understanding of the activation of the innate immune system through pattern recognition receptors (PRRs) is improving the mechanistic understanding of adjuvants, and recent conceptual advances highlight the notion that tissue damage, different forms of cell death, and metabolic and nutrient sensors can all modulate the innate immune system to activate adaptive immunity. Furthermore, recent advances in the use of systems biology to probe the molecular networks driving immune response to vaccines ('systems vaccinology') are revealing mechanistic insights and providing a new paradigm for the vaccine discovery and development process. Here, we review the 'known knowns' and 'known unknowns' of adjuvants, discuss these emerging concepts and highlight how our expanding knowledge about innate immunity and systems vaccinology are revitalizing the science and development of novel adjuvants for use in vaccines against COVID-19 and future pandemics.

An adjuvant is a substance that is added to a vaccine to stimulate and enhance the magnitude and durability of the immune response (BOX 1). The traditional development of new vaccine adjuvants has been described as one of the slowest processes in the history of medicine ${ }^{1}$. For more than seven decades since initial licensure in the 1920s, insoluble aluminium salts (alum) remained the only adjuvant included in licensed products, such as vaccines against hepatitis $B$, diphtheria, tetanus and pertussis or human papilloma virus. However, in the late 1990s, the oil-in-water emulsion adjuvant MF59 was first included in a licensed product in Europe known as Fluad, which is a trivalent inactivated vaccine against seasonal influenza licensed for adults older than 65 years of age. In the 20 years since, four other adjuvants have been included in additional products: AS01 (for the shingles vaccine Shingrix and the malaria vaccine Mosquirix), AS04 (for the hepatitis B vaccine Fendrix and the human papilloma vaccine Cervarix), AS03 (for the pandemic influenza vaccines Pandemrix and Arepanrix) and cytosine phosphoguanosine (CpG) 1018 (for the hepatitis B vaccine Heplisav-B). Although many other adjuvants have demonstrated high potency in preclinical models during this period, most have not yet achieved licensure in humans, often owing to safety or tolerability concerns. Furthermore, despite their widespread use, the molecular mechanisms by which the available adjuvants - including alum, MF59 and the Adjuvant Systems AS0 adjuvants - actually work in humans is not well understood.

Nevertheless, the past two decades have witnessed a revolution in our understanding of how the innate immune system senses microbes, which offers a huge opportunity for additional insights into adjuvant design and development. In the late 1990s, it was discovered that activation of receptors such as Toll-like receptors (TLRs) - which are typically expressed on dendritic cells (DCs) and sense highly conserved pathogen-associated molecular patterns (PAMPs $)^{2-4}$ in microbes - results in activation of DCs, which stimulates antigen-specific $\mathrm{T}$ and $\mathrm{B}$ cell responses ${ }^{5-8}$. These discoveries provided strong experimental evidence for the major conceptual paradigm proposed by Charlie Janeway in 1989: that the innate immune system senses microbes through pattern recognition receptors (PRRs; such as TLRs) that recognize PAMPs and lead to activation of innate immune cells and the ensuing adaptive immune response ${ }^{9}$. In the decade following the discovery of TLRs, other innate PRRs (such as retinoic acid-inducible gene I (RIG-I) and other RNA sensors) ${ }^{10}$, DNA sensors (such as stimulator of interferon genes (STING) protein) ${ }^{11}$, C-type lectins ${ }^{12}$, nucleotide-binding oligomerization domain (NOD)-like receptors (NLRs) and cytosolic receptors such as NLRP3, which activate the inflammasome ${ }^{13,14}$ - were also discovered and shown to impact adaptive 


\section{Box $1 \mid$ The elements of a vaccine}

A vaccine comprises two components: antigens and adjuvants. Antigens typically consist of proteins or carbohydrates derived from the pathogen, against which an adaptive immune response is desired. An adjuvant is a substance that is added to a vaccine to stimulate and enhance the magnitude and durability of the immune response. The word adjuvant (derived from the Latin word 'adjuvare', meaning 'to help') was coined by the French veterinarian Gaston Ramon, who while working at the Pasteur Institute in 1920 discovered that horses vaccinated against diphtheria had stronger antibody titres if they developed inflammatory abscesses at the site of injection ${ }^{234}$. Ramon was able to show that adding various substances, such as breadcrumbs or starch, to the inactivated diphtheria toxin caused inflammation at the injection site and also boosted antibody production in response to the vaccine ${ }^{235}$. At around the same time, the British immunologist Alexander Thomas Glenny, at the Wellcome Physiological Research Laboratory in London, serendipitously discovered the adjuvant properties of alum and, in 1925-1926, developed the alum-precipitated diphtheria toxoid ${ }^{236}$. Glenny et al. were trying to purify diphtheria toxoid (inactive toxin) using potassium aluminium sulfate to develop a vaccine. Surprisingly, vaccines developed using aluminium salt precipitation led to better antibody responses than soluble toxoids. Since then, aluminium-containing adjuvants have been incorporated into billions of doses of vaccines and administered to millions of people every year.

immunity. Molecules that target these receptors are also being pursued as potential vaccine adjuvants ${ }^{15}$.

Despite these major recent advances, our mechanistic understanding of how the currently available adjuvants included in licensed vaccines actually function remains underdeveloped. However, two major advances that have occurred during the past decade are beginning to yield deeper insights into the mechanism of action of adjuvants and are revitalizing the process of adjuvant discovery and development, which has been the status quo for decades. First, immunologists' view of how the immune system can be stimulated has evolved beyond the paradigm of pattern recognition by PAMPs originally proposed by Janeway. Thus, evidence has emerged that DCs and other cells of the innate immune system can become activated not only by pathogen sense via PRRs but also by tissue damage that results in the release of damage-associated molecular patterns (DAMPs) ${ }^{16,17}$ and cell death via necroptosis or pyroptosis ${ }^{18,19}$, as well as other stress signals such as amino acid starvation (via ancient stress and nutrient sensing pathways) $)^{6,20}$. Many of these stimuli have been shown to induce adaptive immunity, thus providing support for the concept of the 'Danger Model' proposed by Polly Matzinger in 1994 (REF. ${ }^{21}$ ). Second, the use of systems biology approaches to probe the innate and adaptive immune responses to vaccines and define the molecular networks that drive innate and adaptive immune response to vaccination in humans is beginning to yield fundamental new mechanistic insights about how immune responses are initiated and controlled ${ }^{22}$. This 'systems vaccinology' approach is now being increasingly used to assess immune responses to vaccination in humans, to identify molecular signatures that can predict vaccine efficacy and to obtain mechanistic insights. In this Review, we discuss how these advances are revitalizing the science of adjuvants. First, we discuss the known facts ('known knowns') and the known gaps ('known unknowns') of the adjuvants in licensed products. Second, we discuss several emerging concepts about the immunological mechanisms of action of adjuvants. In the final section, we provide a conceptual framework in which we highlight how systems-based approaches are beginning to revitalize adjuvant design and development.

\section{Adjuvants in licensed vaccines \\ Endogenous adjuvants in live vaccines}

Although only a handful of adjuvants are available for clinical use, it is now clear that many vaccines that have been used safely in billions of humans contain endogenous adjuvants. Significant conceptual advances in innate immunity and delineation of the critical innate receptors, the PRRs, have led to the realization that many live vaccines that are widely used induce immune responses partly by activating specific PRRs ${ }^{23}$. These vaccines consist of live attenuated pathogens that activate innate immunity through the expression of various PAMPs. For example, the live attenuated yellow fever vaccine (YF-17D) - one of the most potent vaccines ever developed and administered to 600 million people globally - was shown to activate the innate immune system by signalling through multiple TLRs (TLR2, TLR3 and TLR7-TLR9) as well as through RIG-I and melanoma differentiation-associated protein 5 (MDA5) ${ }^{24}$ (FIG. 1). Importantly, TLR signalling is also essential for the immunogenicity of YF-17D ( REFS $^{24,25}$ ), although the additional impact of sustained antigen expression through replication of the attenuated virus should not be underappreciated. Similarly, the Bacillus Calmette-Guérin (BCG) vaccine against tuberculosis, of which more than 4 billion doses have been administered, activates TLR2, TLR4, TLR9 and CD209 antigen (also known as DC-SIGN $)^{26,27}$. In addition, certain inactivated vaccines also trigger TLRs, such as the seasonal influenza vaccines that activate the innate immune system via TLR7 and myeloid differentiation primary response 88 (MyD88) signalling pathways ${ }^{28}$. These findings have further pointed to the continued rational development of TLR agonists as critical targets in adjuvant design, especially in the context of subunit vaccines, which do not contain endogenous adjuvants. Numerous studies have demonstrated the efficacy of synthetic TLR ligands as vaccine adjuvants in mice and in non-human primates (NHPs) in promoting enhanced and more durable antibody responses to vaccination with recombinant protein antigens, virus-like particles or DNA-encoded antigens ${ }^{29,30}$.

The fact that live viral vaccines typically induce robust and persistent antibody responses in humans $\mathrm{s}^{31,32}$ raised the possibility that adjuvants given with inactivated vaccines could induce such robust and persistent antibody responses too. On the basis of the discovery that the yellow fever vaccine engages multiple DC subsets via multiple TLR ligands ${ }^{24}$, we designed synthetic nanoparticles that contain TLR4 and TLR7/TLR8 ligands as adjuvants, which were used in combination with soluble protein antigens, such as chicken ovalbumin or influenza haemagglutinin ${ }^{33}$. Experiments in mice showed that these nanoparticle vaccines induced robust and durable antigen-specific antibody responses, robust and persistent antigen-specific germinal centre (GC) reactions, $\mathrm{T}$ follicular helper $\left(\mathrm{T}_{\mathrm{FH}}\right)$ cell responses and long-lived plasma cells (LLPCs) ${ }^{33}$. Of note, these robust responses induced by the combination of TLR ligands lasted a lifetime in mice and required activation 
a TLRs

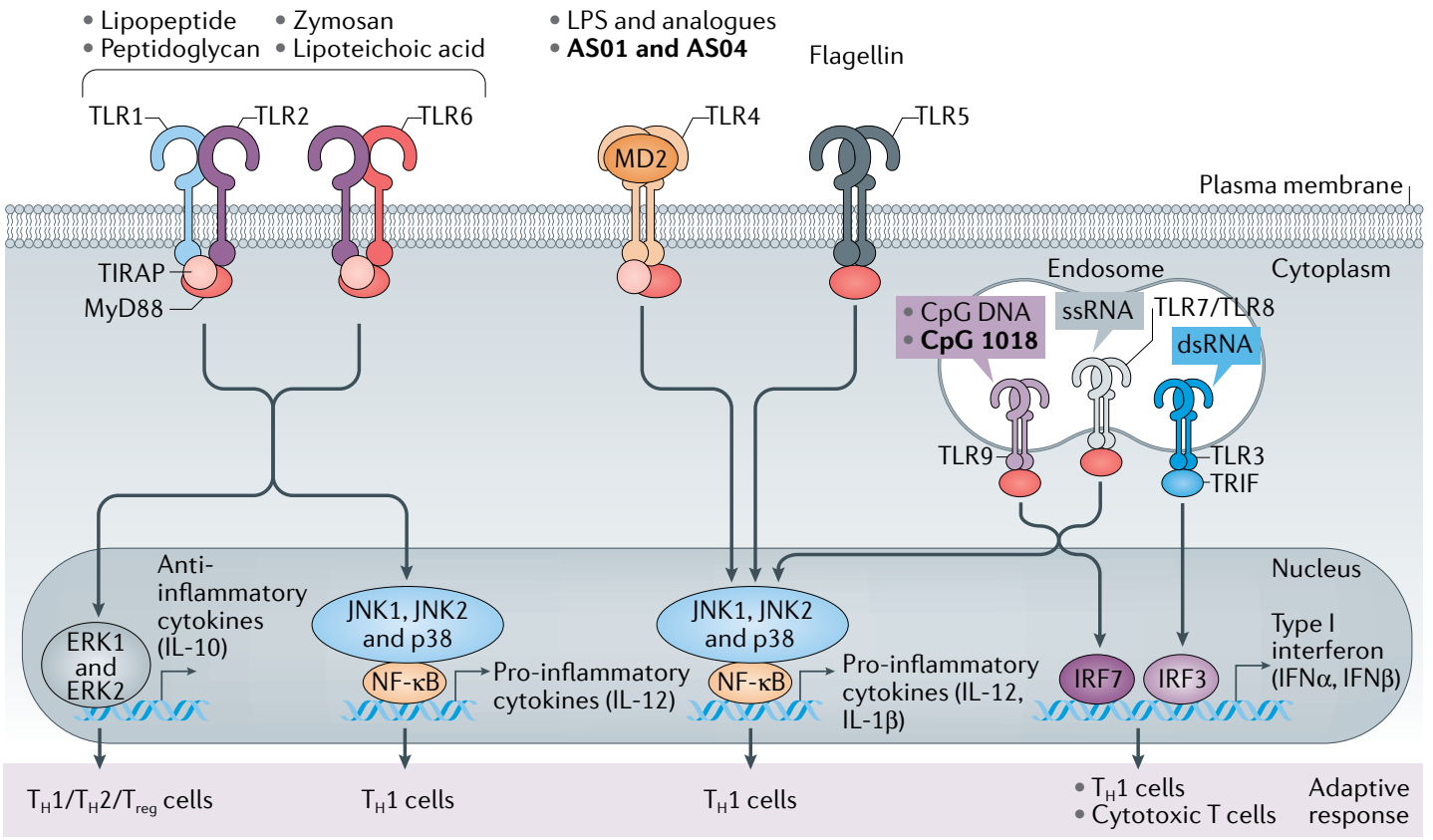

\section{b Cytosolic PRRs}

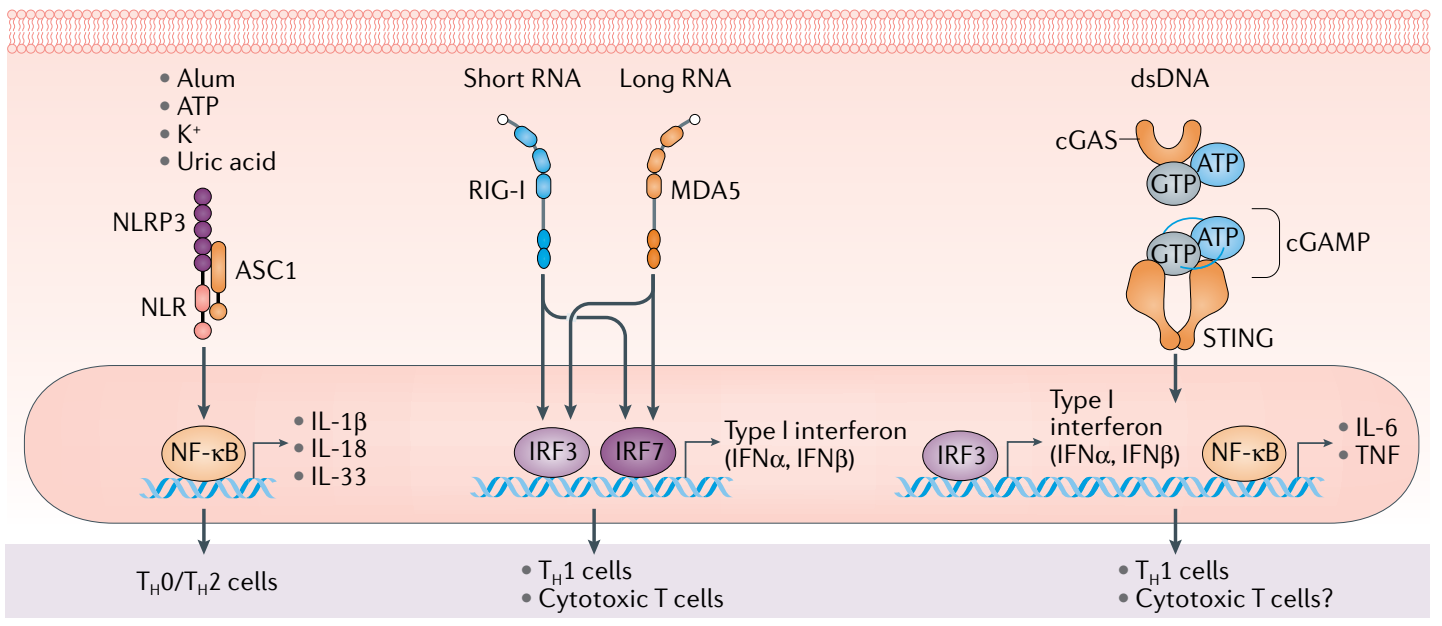

c CLRs

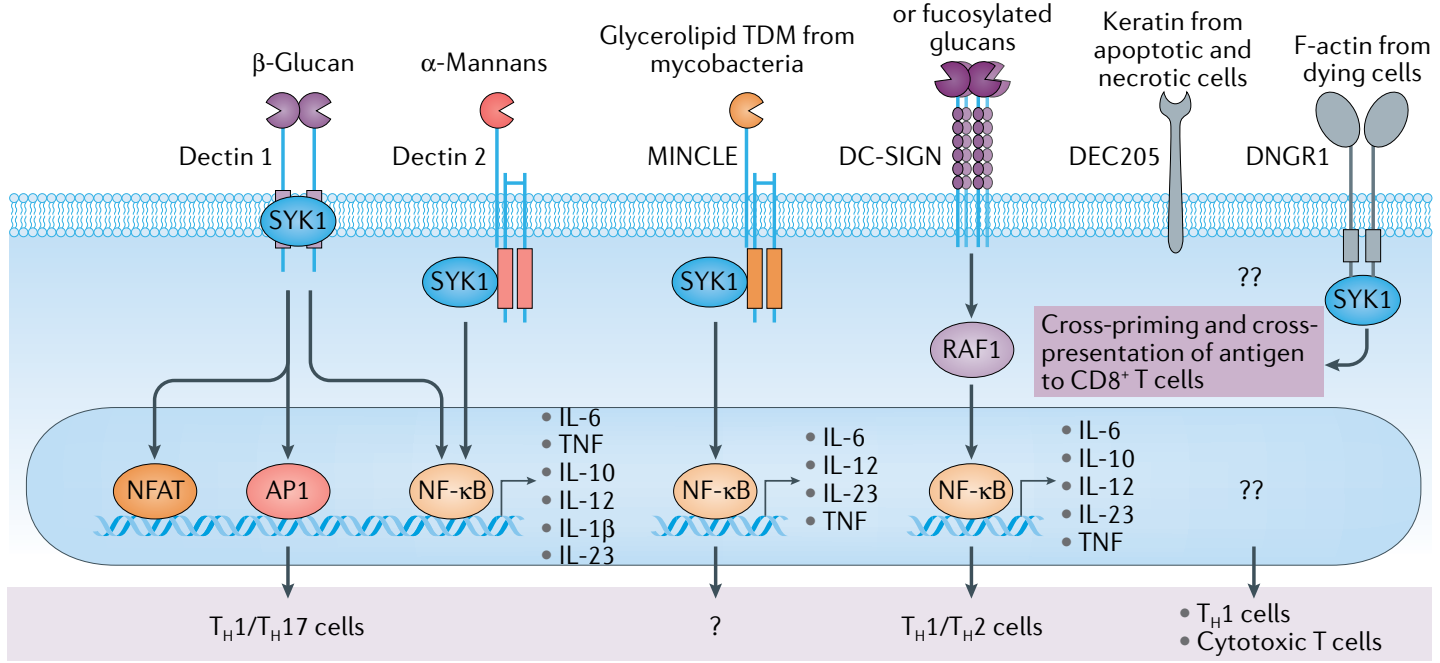


4 Fig. $1 \mid$ Molecular targets of adjuvants. a| Toll-like receptors (TLRs) TLR1, TLR2, TLR4, TLR5 and TLR6 are expressed on the cell surface, whereas TLR3, TLR7, TLR8 and TLR9 are expressed in endosomes. TLR1 and TLR6 heterodimerize with TLR2 and signal through the myeloid differentiation primary response 88 (MyD88) pathway to activate NF- $\mathrm{kB}$ and MAP kinases, leading to secretion of pro-inflammatory and anti-inflammatory cytokines. TLR4 and TLR5 function as homodimers and signal through the MyD88 pathway. TLR7 and TLR9 also use the MyD88 pathway, but rapidly activate IRF7 to induce type I interferons. TLR3 uses TIR domain-containing adapter-inducing IFN $\beta$ (TRIF) signalling to induce type I interferons through IRF3. b | Cytosolic pattern recognition receptors (PRRs) are sensors of pathogen-associated molecular patterns (PAMPs) and damage-associated molecular patterns (DAMPs) present inside the cytoplasm of the cell. Nucleotide-binding oligomerization domain (NOD)-like receptors (NLRs) are cytosolic sensors of bacterial PAMPs but also recognize multiple cellular products including ATP, uric acid and $\mathrm{K}^{+}$to activate the NF- $\kappa B$ pathway and induce cytokines driving T helper $2\left(\mathrm{~T}_{\mathrm{H}} 2\right)$ cell differentiation. Retinoic acid-inducible gene I (RIG-I) and melanoma differentiation-associated protein 5 (MDA5) are intracellular viral sensors that drive type I interferon response through IRF3 and IRF7. The cGAS-stimulator of interferon genes (STING) pathway recognized double-stranded DNA (dsDNA) to induce the NF-kB pathway. $\mathrm{c}$ |C-type lectin receptors (CLRs) are cell surface molecules expressed on multiple myeloid cell subsets. Dectins 1 and 2 and MINCLE recruit SYK1 and activate NF-KB through the CARD9-BCL-10-MALT1 complex. Furthermore, dectin 1 has been shown to induce the NFAT and AP1 pathways in macrophages and dendritic cells (DCs) and in in vitro experimental models, respectively. Dectins are also specialized in inducing antifungal immunity. Dendritic cell-specific ICAM3-grabbing non-integrin 1 (DC-SIGN) activates NF- $\kappa B$ via acetylation of p65; however, the resulting gene expression is poorly understood although IL-10 expression has been shown to be induced. DEC205 and DNGR1 are known to induce cross-presentation but the signalling pathways are unknown. ASC, apoptosis-associated speck-like protein containing a CARD; CpG, cytosine phosphoguanosine; dsRNA, double-stranded RNA; LPS, lipopolysaccharide; ssRNA, single-stranded RNA; TDM, trehalose-6,6-dimycolate; $\mathrm{T}_{\text {reg }}$ cell, regulatory T cell.

Isotype switching

A process in which antigen-experienced B cells switch from making lgM to other isotypes. of multiple DC subsets and direct activation of B cells ${ }^{33}$. Subsequent studies in NHPs showed that such nanoparticle-encapsulated TLR ligands were potent adjuvants in stimulating robust and persistent antibody responses, $\mathrm{GC}$ and $\mathrm{T}_{\mathrm{FH}}$ cell responses and a high frequency of LLPCs that persisted until the termination of the study at 70 weeks $s^{30,34,35}$. It seems that generating LLPCs may be the key to generating durability of antibody responses, and adjuvants driving differentiation of LLPCs will be of particular interest in vaccinology $y^{36,37}$. Thus, developing adjuvants that promote LLPCs represents a major challenge in vaccinology.

Recent work suggests that slow-release delivery systems (such as osmotic pumps) that release the antigen with delayed kinetics can enhance the magnitude, quality and persistence of antibody responses ${ }^{38,39}$. Although this has been attributed to enhanced GC reactions owing to longer antigen retention in lymph nodes, the effect of sustained activation of innate cells by the adjuvants has not been thoroughly evaluated. In this context, we and others have found that 3M-052, a novel TLR7/TLR8 agonist that is released slowly from the site of administration ${ }^{40}$, results in activation of monocytes and DCs that lasts for 3-4 weeks ${ }^{30}$, and induces persistent antibody responses and LLPCs of a much higher magnitude (up to 100-fold greater) than those observed with alum ${ }^{30,35}$ that lasted until the termination of the study at 70 weeks post immunization.

\section{Alum}

Alum is the most commonly used adjuvant and induces antibody responses and $\mathrm{CD} 4^{+} \mathrm{T}$ helper cell responses in humans $\mathrm{s}^{41-43}$ (TABLE 1). These $\mathrm{T}$ helper responses are $\mathrm{T}$ helper $2\left(\mathrm{~T}_{\mathrm{H}} 2\right)$ cell-biased in mice ${ }^{44-47}$, but this bias is less clear in humans ${ }^{48}$. It was long thought that alum mediated its adjuvant effects predominantly through a 'depot effect' mechanism that involved the slow release of antigens from the site of immunization ${ }^{49}$. Consistent with this, chemical modification of antigens with short peptides composed of repeating phosphoserine (pSer) residues enhances binding to alum - which results in prolonged immunogen bioavailability - and greatly enhances GC and antibody responses, compared with conventional alum-adsorbed antigens ${ }^{50}$. In addition, alum actually exerts several effects on the immune system $^{51}$. As discussed above, although TLRs seemed to be essential for the immunogenicity of live viral vaccine such as the yellow fever vaccine YF-17D ${ }^{24,25}$, the immune responses stimulated by immunization with alum plus antigen were unaffected in mice lacking the critical adaptor proteins involved in TLR signalling: MyD88 or TIR domain-containing adapter-inducing IFN $\beta$ (TRIF) ${ }^{52}$. This suggested that the adjuvant effects of alum occurred through a mechanism independent of TLR signalling. Later studies showed that alum activated the NLRP3 inflammasome ${ }^{53-56}$, although the data are contradictory regarding the relative importance of this in mediating alum's adjuvant effect ${ }^{57,58}$. Experiments assessing alum-enhanced immune responses in mice deficient in NLRP3 signalling seem to give conflicting results on whether NLRP3 inflammasome activation is necessary for adaptive immune responses (TABLE 1).

Alum can also enhance adaptive immunity by causing tissue damage that induces uric acid-mediated activation of inflammatory $\mathrm{DCs}^{59}$. Injection of alum rapidly recruited various cells, including neutrophils, which released neutrophil extracellular traps (NETs) composed of chromatin ${ }^{60,61}$. The DNA released in NETs partially mediated the adjuvant activity of alum ${ }^{62,63}$. For example, in mice, immunization with antigen plus alum induced cell death and the subsequent release of host cell DNA, which stimulated an antigen-specific IgE response and $\mathrm{T}_{\mathrm{H}} 2$ cell responses. $\mathrm{T}_{\mathrm{H}} 2$ cell responses were associated with IgE isotype switching via mechanisms dependent on the TANK-binding kinase 1 (TBK1) and the transcription factor IRF3, which are critical components of the signalling pathway mediated by the cytosolic DNA sensor STING $^{62}$ (TABLE 1). In some of those studies, co-injection of DNase preparations with alum and antigen reduced the immune response to vaccination. However, a subsequent study reported that commercial DNase preparations are contaminated with proteases, which were responsible for some of the inhibitory effects of DNase preparations on the adjuvant activity of alum ${ }^{64}$. Nevertheless, DNase lowers responses induced by alum to some extent, but the effect of DNase seemed to be independent of its ability to cleave $\mathrm{DNA}^{64}$. Thus, future work should be aimed at clarifying the relative importance of DNA sensing in mediating the induction of antibody and $\mathrm{CD} 4^{+} \mathrm{T}$ helper cell responses induced by immunization with alum plus antigen. Alum is also used in inactivated virus COVID-19 vaccines that have been approved for limited or emergency use in certain countries ${ }^{65,66}$ (TABLE 2). Hence, the mechanism of action of alum is complex, with likely several factors contributing simultaneously in small animals, and the contribution of the formulation cannot be ignored ${ }^{67}$. 


\section{MF59}

MF59 is an oil-in-water emulsion adjuvant that has been included in a licensed influenza vaccine in Europe since 1997 and has now been administered to more than 100 million people in more than 30 countries. This adjuvant comprises droplets of squalene oil, a biodegradable, biocompatible oil that is a normal component of the human body, stabilized in an aqueous buffer by the surfactants Tween 80 and Span 85 , which are commonly used non-ionic surfactants ${ }^{68}$. Its mechanism of action has been explored and is reviewed elsewhere $^{69}$. To summarize briefly, administration of

\section{Table 1 | The 'known knowns' and 'known unknowns' of adjuvants used in licensed vaccines}

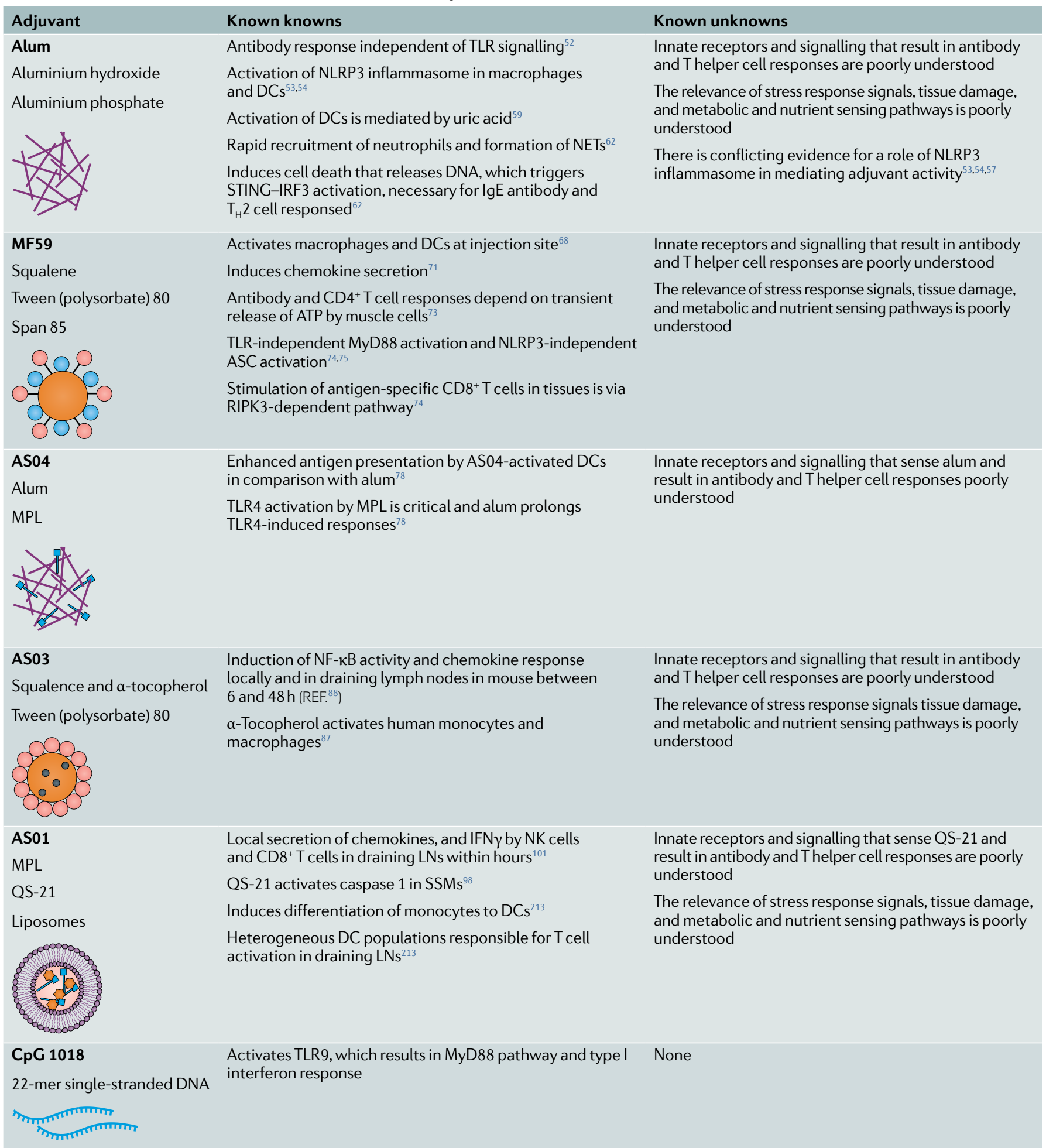

ASC, apoptosis-associated speck-like protein containing a CARD; DC, dendritic cell; IFN $\gamma$, interferon- $\gamma$; LN, lymph node; MPL, 3-O-desacyl-4'-monophosphoryl lipid A; MyD88, myeloid differentiation primary response 88; NET, neutrophil extracellular trap; NK cell, natural killer cell; STING, stimulator of interferon genes; SSM, subcapsular sinus macrophage; $\mathrm{T}_{4} 2$ cell, T helper 2 cell; TLR, Toll-like receptor. 
Table 2 | Vaccine adjuvants in COVID-19 vaccines in limited use or in advanced clinical trials

\begin{tabular}{|c|c|c|c|c|}
\hline Adjuvant & Vaccine & Manufacturers & Status & Refs \\
\hline Alum & Inactivated SARS-CoV-2 virus vaccines & $\begin{array}{l}\text { Sinopharm } \\
\text { Sinovac }\end{array}$ & $\begin{array}{l}\text { Approved for limited } \\
\text { or emergency use in } \\
\text { certain countries }\end{array}$ & 65,66 \\
\hline Matrix-M & $\begin{array}{l}\text { Recombinant SARS-CoV-2 spike (S) } \\
\text { protein }\end{array}$ & Novavax & Phase III & 230 \\
\hline AS03 & $\begin{array}{l}\text { Recombinant SARS-CoV-2 spike (S) } \\
\text { protein as a soluble protein or on } \\
\text { virus-like particles }\end{array}$ & $\begin{array}{l}\text { GSK (AS03) } \\
\text { Sanofi (antigen) } \\
\text { Medicago (antigen) }\end{array}$ & $\begin{array}{l}\text { Phase I/II } \\
\text { Phase III }\end{array}$ & 85,86 \\
\hline CpG 1018 & $\begin{array}{l}\text { Recombinant SARS-CoV-2 spike (S) } \\
\text { protein on virus-like particles }\end{array}$ & $\begin{array}{l}\text { Dynavax (CpG 1018) } \\
\text { Medicago (antigen) }\end{array}$ & Phase I/II & 86 \\
\hline $\begin{array}{l}\text { TLR7/TLR8 ligand } \\
\text { adsorbed in alum }\end{array}$ & Inactivated SARS-CoV-2 vaccines & Bharath Biotech & $\begin{array}{l}\text { Phase III/emergency } \\
\text { use in India }\end{array}$ & 233 \\
\hline
\end{tabular}

COVID-19, coronavirus disease 2019; SARS-CoV-2, severe acute respiratory syndrome coronavirus 2; TLR, Toll-like receptor.

MF59 in muscle activates myeloid cells - such as macrophages and DCs - which respond by producing chemokines such as CC-chemokine ligand 2 (CCL2), CCL4, CCL5 and CXC-chemokine ligand 8 (CXCL8; also known as IL-8), which in turn recruit neutrophils, eosinophils and more monocytes and DCs to the injection site (TABLE 1). These cells amplify the response further and migrate to draining lymph nodes to activate $\mathrm{B}$ and $\mathrm{T}$ cells $\mathrm{s}^{69,70}$. Studies in mice demonstrate that MF59 induces a broader range of cytokines and chemokines than alum, and rapidly recruits $\mathrm{CD} 11 \mathrm{~b}^{+}$inflammatory cells to the site of injection ${ }^{71}$. These observations based on small animal studies have been largely substantiated by similar studies carried out in $\mathrm{NHPs}^{72}$. Intramuscular injection of an MF59-adjuvanted influenza vaccine into mice results in the transient extracellular release of ATP, and local injections of apyrase, an ATP-hydrolysing enzyme, inhibited cell recruitment and antigen-specific $\mathrm{CD}^{+} \mathrm{T}$ cell responses and antibody responses induced by MF59, but not by alum or incomplete Freund's adjuvant ${ }^{73}$. These data demonstrate that transient release of ATP induced by MF59-adjuvanted vaccines mediates the adaptive immune response.

With regards to the innate immune receptors involved in sensing MF59, Seubert et al. demonstrated that MF59-adjuvanted antibody responses were dependent on MyD88, but independent of NLRP3 inflammasome activation $^{74}$. Immunization of Myd88-knockout mice with an MF59-adjuvanted vaccine against Neisseria meningitidis (rMenB) resulted in lower antibody titres compared with those obtained in control mice ${ }^{74}$. Surprisingly, however, MF59 did not activate TLR signalling in HEK293 cell lines expressing TLRs, which led the authors to conclude that MF59 induces antibody responses via an MyD88-dependent mechanism that is independent of $\mathrm{TLRs}^{74}$. In an independent study, immunization of an MF59-adjuvanted H5N1 subunit vaccine into mice genetically deficient in apoptosis-associated speck-like protein containing a CARD (ASC), an adaptor protein within the NLRP3 inflammasome, resulted in reduced $\mathrm{H} 5$-specific IgG antibody titres relative to that observed in wild-type mice ${ }^{75}$. Interestingly, and consistent with the study by Seubert et al., the response was intact in
Nlrp3-knockout and Casp1-knockout mice ${ }^{75}$, which led the authors to conclude that MF59-adjuvanted vaccines induce antibody responses via an ASC-dependent, but inflammasome-independent, pathway. Finally, our recent work demonstrates that subcutaneous immunization of mice with MF59 plus antigen stimulates antigen-specific $\mathrm{CD}^{+} \mathrm{T}$ cell responses (which are stimulated at very low magnitude in the draining lymph nodes, but can be detected at much higher frequencies in tissues such as the lung and liver), via a mechanism dependent on receptor-interacting serine/threonine protein kinase 3 (RIPK3), which is a key mediator of necroptosis ${ }^{76}$. Thus, immunization of mice with antigen mixed with MF59 or its mimetic Addavax, but not with alum, induced rapid RIPK3-dependent necroptosis of lymph node macrophages. RIPK3-deficient mice were impaired in their capacity to mount antigen-specific $\mathrm{CD}^{+} \mathrm{T}$ cell responses in the lung and liver, in response to subcutaneous immunization with antigen plus Addavax. However, surprisingly, such responses were normal in mice deficient in mixed-lineage kinase domain-like protein (MLKL), a downstream mediator of necroptosis, suggesting that the impaired $\mathrm{CD} 8^{+} \mathrm{T}$ cell responses observed in RIPK3-deficient mice occurred through a mechanism independent of necroptosis ${ }^{76}$. In contrast to the effects observed on $\mathrm{CD}^{+} \mathrm{T}$ cells, antibody responses were not affected in RIPK3-deficient mice or in caspase 1-deficient mice. However, they were impaired by administration of the pan-caspase inhibitor Z-VAD-FMK, suggesting a contribution of apoptotic caspases to the induction of antibody responses. These observations in mice provide mechanistic insights into the adjuvant effects of squalene-based adjuvants such as MF59, but the extent to which such mechanisms operate in humans remains to be determined.

\section{The ASO Adjuvant Systems}

The Adjuvant Systems AS0 have been developed by GlaxoSmithKline over the past three decades and are based on a rational combination of classical adjuvant molecules - including alum, emulsions and liposomes - to obtain maximal adjuvant effect with acceptable tolerability, in combination with immunostimulatory molecules, such as TLR ligands and others. 
AS04. AS04 consists of 3-O-desacyl-4'-monophosphoryl lipid A (MPL), a detoxified form of lipopolysaccharide (LPS) extracted from Salmonella minnesota, which is adsorbed on aluminium salts. Importantly, studies in mice have revealed that MPL retains its complete immunostimulatory activity through TLR4 activation, when adsorbed on alum. The adjuvant effect of AS04 is mediated by signalling through TLR4 on innate cells, in combination with the inherent immunomodulatory properties of alum ${ }^{77}$ (TABLE 1). Addition of AS04 to the hepatitis B virus (HBV) and the human papillomavirus (HPV) vaccines based on recombinant antigens induced higher levels of antibodies in comparison with the same antigens adjuvanted with just alum, demonstrating the added value of the TLR4 agonist MPL in humans ${ }^{78-81}$. The higher immunogenicity also translated into a high and long-lasting efficacy of the HPV-16/18 vaccine. Data available now show that the efficacy of the vaccine approaches can reach $100 \%$, more than 9 years after primary immunization ${ }^{80,82}$. Further studies are required to better understand the precise mechanisms behind this beneficial effect of the AS04-adjuvanted HPV vaccine. For example, the inclusion of AS04 results in a greater breadth of immune response against diverse HPV strains in humans, through mechanisms that are not yet clearly defined $^{83}$.

AS03. AS03 is a squalene oil-in-water emulsion adjuvant that is similar to MF59, but also contains $\alpha$-tocopherol (vitamin E) as an additional immune-enhancing component. AS03 is licensed in combination with pandemic influenza vaccines ${ }^{84}$ (TABLE 1). AS03 is being evaluated in clinical trials as a vaccine adjuvant for numerous different recombinant spike protein COVID-19 vaccines ${ }^{85,86}$ (TABLE 2). AS03 enhances the magnitude and breadth of antibody responses and $\mathrm{CD} 4^{+} \mathrm{T}$ cell responses, leading to enhanced protection against flu compared with non-adjuvanted vaccines ${ }^{87}$. Mouse ${ }^{88,89}$ and human ${ }^{90,91}$ studies have demonstrated that AS03 triggers transient innate immune responses, although additional mechanisms of action are likely also involved. In mice, AS03 stimulated a transient production of cytokines at the site of injection and in the draining lymph nodes ${ }^{88}$. $\alpha$-Tocopherol modulated the expression of certain chemokines and cytokines such as CCL2, CCL3, IL-6, granulocyte colony-stimulating factor (G-CSF; encoded by CSF3) and CXCL1, enhanced antigen uptake in monocytes and increased the recruitment of granulocytes to the draining lymph nodes ${ }^{88}$. A subsequent study in mice demonstrated that intramuscular injection of AS03 elicited a rapid and transient downregulation of genes involved in lipid metabolism in the draining lymph nodes. In vitro, in myeloid cell lines, these changes were associated with changes in lipid composition and altered endoplasmic reticulum (ER) morphology, and with activation of the unfolded protein response (UPR) pathway. In vivo, treatment with a chemical inhibitor of the ER stress response or genetic deletion in myeloid cells of the ER stress sensor kinase IRE1 $\alpha$ reduced IL- 6 production ${ }^{92}$. In humans, within $24 \mathrm{~h}$ of vaccination with AS03-adjuvanted H5N1 avian influenza vaccine, there were increased serum levels of
IL-6 and IP10 (also known as CXCL10) as well as transcriptional signatures of interferon signalling and antigen processing and presentation in DCs, monocytes and neutrophils ${ }^{90}$. Interestingly, this early upregulation of interferon signalling was found to predict antibody titres at 56 days $^{90}$. Given the similarity of AS03 and MF59 both being squalene-based adjuvants, it is likely that they engage common pathways of innate immune activation. As described, both adjuvants stimulate innate cytokines and chemokines, but the extent to which they both depend on the IRE1 $\alpha$ and RIPK3-dependent pathways ${ }^{76}$ remains to be determined.

AS01. AS01 is included in a licensed vaccine against varicella zoster (Shingrix), approved for use in older adults (50 years and older), with high efficacy $(97.2 \%)^{93}$. The adjuvant AS01, which is a unique combination of two different immunostimulatory components the TLR4 ligand used in AS04 (MPL) and an isolated and purified saponin fraction (QS-21) - is also used in a licensed malaria vaccine, which is currently being implemented in Africa in a limited campaign ${ }^{94}$. QS-21 is a triterpene glycoside purified from the bark extracts of the tree Quillaja saponaria Molina. Preclinical studies using the QS-21 adjuvant showed enhanced antibody as well as cell-mediated immune responses ${ }^{95-97}$. However, although QS-21 alone was potent, there were significant concerns about its tolerability profile when used as a single-component adjuvant in human vaccines. In AS01, MPL and QS-21 are formulated together in liposomes in the presence of cholesterol, which is used to bind QS-21 into the liposome and to quench its reactogenicity. The MPL activates the innate immune system through TLR4, largely through TRIF-dependent signalling. In addition, studies in mice show that QS-21 activates caspase 1 in subcapsular sinus macrophages (SSMs) in the draining lymph node ${ }^{98}$ (TABLE 1). Although caspase 1 activation by QS-21 is NLRP3-dependent in vitro, NLRP3 does not seem to have a role in adjuvanticity in vivo ${ }^{98,99}$. When formulated in liposomes, QS-21 enters the cells through cholesterol-dependent endocytosis and induces lysosomal destabilization, followed by tyrosine-protein kinase SYK activation ${ }^{100}$. Collectively, the combination of the specific activation of different innate pathways by the two individual molecules is critical for the full adjuvant effect of AS01, as depletion of TLR4, caspase 1 or SSMs individually impairs the adjuvant effect in mouse models. Hence, a striking feature of AS01 is the synergy that occurs to induce novel pathways that are not triggered by either component alone ${ }^{98,101}$. The key emergent pathways seem to be IFN $\gamma$-related, as blocking IFN $\gamma$ in vivo abrogates the synergistic effect of MPL and QS-21. The typical immune response to AS01 adjuvant is characterized by an increase in polyfunctional $\mathrm{CD} 4^{+} \mathrm{T}$ cells (such as T cells that express IL-2, IFN $\gamma$ and TNF) specific to the co-administered antigen, along with enhanced functional antibodies ${ }^{101}$. In summary, in AS01, two well-established adjuvant molecules were combined in a novel delivery system (liposome) that resulted in a synergistic engagement of innate immunity, such that the adaptive immune response induced was greater than the individual sum of the independent components. 
Immunosenescence

Ageing of the immune system.

CpG-B class oligonucleotide An oligonucleotide containing $\mathrm{CpG}$ dinucleotides that directly induce B cell proliferation.
Nevertheless, although much has been learned in preclinical models, there is still much to be learned about how AS01 functions in humans, particularly in older subjects, in which it seems to have a remarkable ability, amongst licensed adjuvants, to overcome 'immunosenescence'.

Taken together, the Adjuvant Systems AS0 exert their effects by multiple mechanisms, depending on which components were used in the formulation. Using a combination of adjuvants that were already in various phases of preclinical or clinical testing, rational combinations were created to maximize potency, while ensuring that an acceptable tolerability and safety profile were also in place to enable successful product development. Nevertheless, there was a long, arduous and challenging path to licensure, which we hope to abbreviate in the future, on the basis of the lessons learned. We believe that key lessons will continue to emerge from human studies using systems biology approaches, particularly those that are focused on challenging the assumptions on mechanisms of action, which have emerged from small animal studies. We also believe that key observations will come from mechanism-based studies in large animal models, which allow more comprehensive analysis, but observations from selective small and large animal studies will still need to be substantiated in humans ${ }^{102}$.

\section{Cytosine phosphoguanosine 1018}

There are three classes of $\mathrm{CpG}$ oligonucleotide ligands for TLR9, which can be distinguished by different nucleotide sequence motifs and their capacity to stimulate IFNa in plasmacytoid DCs ${ }^{103}$ (TABLE 1). The TLR9 agonist CpG 1018, a 22-mer unmethylated CpG-B class oligonucleotide, is a potent $\mathrm{T}_{\mathrm{H}} 1$ cell adjuvant and stimulates strong B cell and NK cell activation. CpG 1018 is currently being evaluated in clinical trials as a potential vaccine adjuvant for COVID-19 vaccines ${ }^{86}$ (TABLE 2). Furthermore, this molecule is a component of Heplisav- $\mathrm{B}$, an improved $\mathrm{HBV}$ vaccine licensed for use in adults (age $>18$ years). Although there are other HBV vaccines that were already licensed and used widely, they are typically administered in a three-dose regimen, whereas the key advantage of Heplisav- $B$ is that it offers a simplified two-dose regimen ${ }^{104}$. In preclinical studies in mice, CpG nucleotides induced significantly higher antibody responses to various antigens, including the surface antigen of HBV, HBsAg, compared with unadjuvanted or alum-adjuvanted formulations $s^{105}$. However, the expression pattern of TLR9 in mice is different from that in humans and macaques. In mice, many cell types, including cells of monocyte/macrophage lineage, express TLR9, whereas in humans and macaques TLR9 expression is strictly limited to plasmacytoid DCs and B cells, which highlights the importance of understanding the molecular mechanisms of action of novel adjuvants in humans ${ }^{106}$. Finally, the different immunostimulatory capacities of the distinct classes of $\mathrm{CpG}$ molecules mentioned above seem to depend on whether they occur in monomeric or multimeric forms. Thus, monomeric CpG-B oligonucleotides localize to lysosome-associated membrane protein 1 (LAMP1)-positive endosomes and promote plasmacytoid DC maturation but little or no IFNa production; however, when complexed into microparticles, CpG-B localizes to transferrin receptor 1 (TFR1)-positive endosomes and leads to IFNa production, via a TLR9-dependent mechanism ${ }^{103}$. Furthermore, monomeric CpG-B can be readily taken up by $B$ cells and stimulate their activation $^{107}$. By contrast, CpG-A, which spontaneously forms nanoparticle-like complexes because of its palindromic structure, also localizes to TFR $1^{+}$endosomes in plasmacytoid DCs and stimulates IFNa production but cannot be taken up by B cells, which do not internalize larger DNA complexes such as $\mathrm{CpG}-\mathrm{A}^{103,107}$. The innate mechanisms and cell types that stimulate adaptive immunity in response to immunization with $\mathrm{CpG}$ plus antigen also seem to depend on the form of CpG. Thus, immunization of mice with soluble CpG-B plus antigen induces $\mathrm{T}_{\mathrm{H}} 1 \mathrm{CD}^{+} \mathrm{T}$ cells and antibody responses through a mechanism dependent on TLR9-mediated MyD88 activation in DCs ${ }^{108}$. Conversely, immunization with an aggregated form of CpG-B plus antigen seems not to depend critically on MyD88 signalling in DCs, arguing for a role of other innate cells in sensing aggregated forms of $\mathrm{CpG}$ and eliciting adaptive immunity ${ }^{108}$.

\section{Emerging concepts in adjuvant biology Role of adjuvant in $\mathrm{CD8}^{+} \mathrm{T}$ cell response}

Although it is well established that adjuvants can enhance antibody responses to vaccination in humans, to date no adjuvant has been shown to induce the magnitude of antigen-specific CD8 ${ }^{+} \mathrm{T}$ cell responses stimulated by live viral vaccines such as YF-17D (REF. ${ }^{109}$ ). It has been argued that the initial dose and persistence of antigen are the dominant determinants of $\mathrm{CD}^{+} \mathrm{T}$ cell responses ${ }^{109}$. However, analysis of the immune responses induced by live viral vaccines in humans has revealed that both the antigen load and the adjuvant signals play critical parts in inducing adaptive immune responses.

In mice, several immunogens in combination with a range of adjuvants induce potent $\mathrm{CD} 8^{+} \mathrm{T}$ cell responses. By contrast, in humans, only live viral vaccines such as yellow fever and smallpox induce a very high magnitude of antigen-specific effector $\mathrm{CD} 8^{+} \mathrm{T}$ cell responses and memory $\mathrm{CD}^{+} \mathrm{T}$ cell responses ${ }^{25,109,110}$. As these are replicating vaccines, these studies have highlighted that the initial viral load (antigen) determines the magnitude of the $\mathrm{CD}^{+} \mathrm{T}$ cell response in humans ${ }^{109}$. Experiments in mice with YF-17D have shown that the activation of DCs, via multiple TLRs and the resulting MyD88 signalling, drives the induction of $\mathrm{CD}^{+} \mathrm{T}$ cells ${ }^{24}$. In addition, YF-17D signals through the RNA sensors RIG-I and MDA5 (REF. ${ }^{25}$ ). Thus, an important question to address is whether there are adjuvants that can mimic the potency of live vaccines in inducing $\mathrm{CD}^{+} \mathrm{T}$ cell responses in humans. Accumulated experience shows that subunit vaccines, even when delivered with potent adjuvants, do not induce $\mathrm{CD}^{+} \mathrm{T}$ cell responses in humans, at least as measured in the blood. However, TLR ligands or combinations of TLR ligands delivered with antigen can induce antigen-specific $\mathrm{CD}^{+} \mathrm{T}$ cell responses in mice ${ }^{111-113}$. Antigen-specific $\mathrm{CD} 8^{+} \mathrm{T}$ cells can also be induced in mice by targeting C-type lectin receptors (CLRs) such as DEC205 (REFS ${ }^{114,115}$ ) to DCs, to promote cross-presentation of antigen to $\mathrm{CD}^{+} \mathrm{T}$ cells. These 


\section{Box 2 | Frontiers in adjuvant design and development}

Recent advances in immunology have highlighted novel cellular and molecular targets for adjuvant development.

- Adjuvants that induce $C D 8^{+} T$ cells and tissue resident memory $T$ cells $\left(T_{R M}\right.$ cells): developing novel adjuvants that induce a high magnitude of antigen-specific CD8 ${ }^{+}$ $T$ cells and $T_{R M}$ cells in humans, similar to that induced by live viral vectors such as the yellow fever vaccine ${ }^{109}$.

- Adjuvants that target non-Toll-like receptor (TLR) pattern recognition receptors (PRRs): developing novel adjuvants that target PRRs other than TLRs, including nucleic acid-sensing receptors such as retinoic acid-inducible gene I (RIG-I) $)^{137}$, stimulator of interferon genes (STING) protein ${ }^{139}$, C-type lectin receptors (CLRs) ${ }^{13}$ and nucleotide-binding oligomerization domain (NOD)-like receptors (NLRs) ${ }^{237}$.

- Metabolic adjuvants: nutrient sensors such as mTOR and GCN2 have key roles in modulating dendritic cell (DC) function and adaptive immunity ${ }^{6,155-160}$. Small molecules that target such metabolic regulators in DCs may represent novel adjuvants.

- Cell death adjuvants: it is now known that uptake of dead cells by DCs can result in cross-presentation of cellular corpse-derived antigens to $C D 8^{+} T$ cells ${ }^{148}$. In particular, recent work has highlighted that receptor-interacting serine/threonine protein kinase 3 (RIPK3)-mediated necroptosis can synergize with NF-kB-dependent inflammation to promote $\mathrm{CD} 8^{+} \mathrm{T}$ cell responses. Furthermore, recent work shows that the adjuvant effects of MF59 in promoting CD8 ${ }^{+} \mathrm{T}$ cell response are largely mediated by the key mediator of necroptosis RIPK3 but via a necroptosis-independent pathway ${ }^{76}$.

- Epigenetic adjuvants: recent work suggests that innate cells including monocytes and macrophages undergo epigenetic modifications and acquire memory-like characteristics following stimulation with pathogen-associated molecular patterns (PAMPs) ${ }^{161-166}$. Thus, small molecules that target such cells may represent epigenetic adjuvants that stimulate heightened activation of particular facets of the innate response (such as an antiviral state, including interferon stimulated genes (ISGs)). Such an epigenetically reprogrammed antiviral state will provide resistance to a broad array of viruses, for some period of time, perhaps a few weeks or so.

strategies have been successful in mice, but it continues to be challenging to translate these outcomes to NHPs or humans ${ }^{29,30,34,116-118}$, despite the observation that the generation of $\mathrm{CD}^{+} \mathrm{T}$ cell responses can be enhanced by the physical linking of TLR ligands to soluble antigens ${ }^{117,118}$. Furthermore, the currently available adjuvants induce much lower magnitude $\mathrm{CD} 8^{+} \mathrm{T}$ cell responses relative to viral vaccines such as YF-17D or smallpox ${ }^{109,110}$, although $\mathrm{CD} 4^{+} \mathrm{T}$ cell responses are more readily induced by subunit vaccines ${ }^{119,120}$. DNA-based vaccines and recombinant viral vectors have been tested extensively in the form of prime-boost regimens ${ }^{121-125}$, but still do not induce the magnitude of $\mathrm{CD}^{+} \mathrm{T}$ cell responses observed with live viral vectors. Therefore, the discovery of adjuvants that induce potent cell-mediated immune responses to subunit vaccines remains a challenge for the future. In this context, it should be noted that recent results from the COVID-19 vaccine trials with mRNA vaccines have demonstrated a high magnitude of $\mathrm{CD} 8^{+} \mathrm{T}$ cell responses in humans ${ }^{126}$. Thus, in the phase I/II trial of the mRNA vaccine from BioNTech/Pfizer, the mean number of IFN $\gamma$-producing $\mathrm{CD}^{+} \mathrm{T}$ cells and $\mathrm{CD}^{+} \mathrm{T}$ cells specific for the SARS-CoV-2 receptor binding domain (RBD) spike protein was approximately 700 and 400 per million peripheral blood mononuclear cells (PBMCs), with high responders having as many as 4,000 spots per million PBMCs. Consistent with this, by intracellular cytokine staining, the frequency of RBD-specific $\mathrm{CD}{ }^{+} \mathrm{T}$ cells was remarkably high, with several vaccinees having frequencies as high as $4 \% \mathrm{RBD}$-specific $\mathrm{CD} 8^{+} \mathrm{T}$ cells of the total
T cell pool ${ }^{126}$. The mechanistic basis of this high magnitude of $\mathrm{CD}^{+} \mathrm{T}$ cell response induced by the mRNA vaccine is unclear, but could be due to activation of the innate immune system by nucleic acid sensing receptors, or other mechanisms, as well as antigen persistence for some period, perhaps a few days or a week or so.

Finally, there is now heightened interest in tissue resident memory $\mathrm{T}$ cells $\left(\mathrm{T}_{\mathrm{RM}}\right.$ cells), which are generally believed to reside permanently within tissues and provide a frontline defence against pathogens ${ }^{127,128}$. $\mathrm{T}_{\mathrm{RM}}$ cells are positioned to rapidly respond upon reinfection at barrier sites. Upon pathogen entry to a mucosal site, $\mathrm{T}_{\mathrm{RM}}$ cells that recognize the pathogen are rapidly activated and stimulate neighbouring cells in the local microenvironment to activate and recruit immune cells and establish an antiviral state. Our recent studies in NHPs suggest that vaccination-induced $\mathrm{T}_{\mathrm{RM}}$ cells in mucosal tissues, upon reactivation, induce an antiviral gene expression programme in resident myeloid cells and $\mathrm{CD} 4^{+} \mathrm{T}$ cells. Of note, the $\mathrm{T}_{\mathrm{RM}}$ cell innate crosstalk significantly reduced the neutralizing antibody titres required for protection against mucosal simian-human immunodeficiency virus (SHIV) challenge ${ }^{129}$. Therefore, a major challenge for adjuvant research is developing adjuvants that can induce a high magnitude of $\mathrm{T}_{\mathrm{RM}}$ cells (BOX 2).

Collectively, these observations suggest that induction of effective $\mathrm{CD}^{+} \mathrm{T}$ cell responses in humans requires the optimal adjuvant signalling, in conjunction with the sustained presence of antigen, at least for a few days. Ideally, the kinetics of antigen presence and its distribution could be designed to be similar to that observed following administration of live viral vaccines. For example, the lessons from analysis of the immune responses to the yellow fever vaccine YF-17D suggest that activation of diverse innate immune receptors (TLRs, RIG-I, or MDA5) is necessary for induction of $\mathrm{CD}^{+} \mathrm{T}$ cell responses. In addition, sustained antigen presence for a week or so, as might be expected to occur during an acute viral infection, could also promote enhanced CD8 ${ }^{+}$ $\mathrm{T}$ cell responses. Hence, the failure of adjuvants to induce $\mathrm{CD}^{+} \mathrm{T}$ cell responses to inactivated vaccines in humans suggests that the antigen load, persistence and distribution needs to be better controlled. It is known that live replicating viral vaccines, such as YF-17D and smallpox, induce an acute viral infection in humans ${ }^{109}$, which conceivably facilitates priming of $\mathrm{CD}^{+} \mathrm{T}$ cells at multiple sites throughout the body, thereby contributing to a greater magnitude of $\mathrm{CD}^{+} \mathrm{T}$ cell response. Therefore, future research should be aimed at better defining the parameters of antigen and adjuvant persistence and distribution and the optimization of such parameters to determine the potential for effective generation of $\mathrm{CD}^{+}$ $\mathrm{T}$ cell response, including $\mathrm{T}_{\mathrm{RM}}$ cells, in humans.

\section{It is not just about DCs}

DCs have long been considered the primary cellular targets of vaccine adjuvants ${ }^{5,68,23,130,131}$. Indeed, there is clear evidence that DCs are essential for the adjuvant activity of TLR ligands $\mathrm{s}^{23}$. Thus, in mice deficient in DCs, the adjuvant activity of TLR ligands is severely compromised ${ }^{33,108}$. Even for non-TLR-based adjuvants such as alum or MF59, conditional deletion of DCs or subsets of DCs 
results in a major impairment of adaptive immune responses $^{59,76}$. These studies have unambiguously placed DCs as one of the key cell types in sensing adjuvants and tuning adaptive immunity. However, the immune system is a complex network of interacting cell types, and although DCs play a central part in innate sensing and orchestrating immune responses, emerging evidence highlights key roles for other cell types in this process. For example, as described previously, the administration of alum and MF59 induces transcriptional changes in muscle cells ${ }^{71}$, more so than in DCs. In addition, ATP release from muscle cells induced by MF59 is critical for its adjuvant activity ${ }^{73}$. Furthermore, monocytes and granulocytes are more readily detected at the site of administration in muscle in response to the chemokine signal induced by the adjuvant ${ }^{70}$, and respond to in vitro stimulation more effectively ${ }^{132}$. In addition, direct stimulation of B cells seems to be an equally important factor in generating long-lasting plasma cell responses ${ }^{33}$. Thus, chimeric mice in which TLR signalling is selectively absent in B cells are severely impaired in their capacity to produce antibodies in response to immunization with TLR ligands plus antigen ${ }^{33}$. Consistent with this, B cell-intrinsic MyD88 signalling was shown to be essential to stimulate antigen-specific B cell and antibody response to a virus-like particle ${ }^{133}$. Lymph node resident macrophages also have central roles in the adjuvanticity of MF59 and QS-21 (REFS ${ }^{98,134}$ ). Finally, few studies have examined the effect of adjuvants on epithelial cells, even though these cells frequently represent the first contact of an external stimulus ${ }^{135}$. Therefore, future work should be aimed at a more comprehensive evaluation of the different cell types involved in the innate sensing of adjuvants, and the mechanisms by which they orchestrate the adaptive immune response.

\section{It is not just about TLRs either}

Although the major focus of adjuvant discovery during the past decade has been to target the TLR pathway, it is now clear that other PRRs can be targeted to achieve an adjuvant effect. The available receptors for potential exploitation by new-generation adjuvants include NLRs $^{136}$, RIG-I-like receptors (RLRs) ${ }^{137}$, CLRs $^{138}$ and STING ligands ${ }^{139}$. Of note, signalling through most of the PRRs, including TLRs, induces some local tissue and cellular damage, and the DAMPs released by this seem to be a critical component of the adjuvant activity of several adjuvants. For instance, NLRs, the cytosolic sensors of bacterial PAMPs, recognize multiple cellular products, including ATP, uric acid and $\mathrm{K}^{+}$efflux, suggesting that this activation is potentially mediated by cellular damage ${ }^{140,141}$ (FIG. 1).

Ligands of STING and RIG-I pathways are thought to be potential adjuvants that stimulate robust $\mathrm{CD} 8^{+}$ $\mathrm{T}$ cell responses in mice. For example, a recent study described the induction of potent $\mathrm{CD}^{+} \mathrm{T}$ cells in mice by $2^{\prime}, 3^{\prime}$-cGAMP, but of particular interest was the significant tumour regression seen with $2^{\prime}, 3^{\prime}$-cGAMP ${ }^{142}$. Stimulation of RIG-I and RLRs offers another potential mechanism to be explored to possibly induce $\mathrm{CD}^{+} \mathrm{T}$ cell responses ${ }^{143,144}$. Chen et al. recently showed that circular RNA activates RIG-I in vivo and can be used as an adjuvant to induce cell-mediated as well as antibody responses ${ }^{145}$. It will be interesting to further evaluate whether circular RNA encoding proteins could induce stronger and more potent $\mathrm{CD}^{+} \mathrm{T}$ cell responses as the expressed antigen is intracellular for efficient presentation by major histocompatibility complex (MHC) class I.

\section{Metabolism, cell death and epigenetics}

As mentioned above, it has become increasingly clear over the past decade that the innate immune system can not only sense microbes via PRRs but is also capable of sensing various kinds of tissue damage and stress signals ${ }^{6,20}$. For example, tissue damage can be caused by trauma, autoimmunity or infections and lead to cell death and the extracellular release of a plethora of DAMPs, such as ATP or uric acid, or fragments of DNA or RNA or high mobility group box 1 (HMGB1), which can activate DCs to stimulate adaptive immunity ${ }^{16,17,146,147}$. This has raised the question of whether these pathways of innate activation can be harnessed in designing novel adjuvants. Indeed, there is emerging evidence that adjuvants that are already in use in the clinic may indeed stimulate immune responses via such pathways (FIG. 2a). For example, as discussed above, there is evidence that, in mice, alum causes cell death and subsequent release of host cell DNA that acts as a DAMP in stimulating immune responses ${ }^{62}$. In addition, as mentioned above, injection of MF59-adjuvanted vaccines into mice stimulates the extracellular release of ATP, whose inhibition by local injections of apyrase diminishes the immune response $^{73}$.

Cell death adjuvants. Cell death has also emerged as a key regulator of immune responses ${ }^{19}$. Early work by Bevan and colleagues showed that exogenous antigens, which normally would not be expected to gain access to the cytoplasm of DCs, could be translocated to the cytoplasm, where they could be processed or presented on MHC class I to stimulate CD8 ${ }^{+} \mathrm{T}$ cell immunity ${ }^{148}$. Subsequent work by many laboratories helped establish this pathway of antigen presentation by DCs and demonstrated that DCs could also acquire antigens from apoptotic cells cross-presenting these to CD8 ${ }^{+}$ $\mathrm{T}$ cells ${ }^{149}$. However, research during the past decade has revealed multiple ways in which cells can die, and much effort has focused on understanding mechanisms of necrosis such as necroptosis and pyroptosis ${ }^{150}$. Necroptosis is mediated by RIPK1, which autophosphorylates and then recruits RIPK3, the kinase activity of which mediates necroptosis ${ }^{151-153}$. The coordinated action of the RIPK1-dependent cell death pathway and NF- $\kappa \mathrm{B}$-dependent inflammation synergize to promote enhanced cross-priming of CD8 ${ }^{+} \mathrm{T}_{\text {cells }}{ }^{154}$. Interestingly, immunization of mice with MF59 plus antigen results in necroptosis of lymph node macrophages and stimulation of antigen-specific $\mathrm{CD}^{+} \mathrm{T}$ cells (present at very low magnitude in the draining lymph nodes but detected at much higher frequencies in the lung and liver $)^{76}$. Interestingly, the RIPK3-mediated stimulation of $\mathrm{CD}^{+} \mathrm{T}$ cells occurred through a mechanism independent of MLKL, which suggests that RIPK3 mediates activation of $\mathrm{CD}^{+}$ $\mathrm{T}$ cells through a necroptosis-independent mechanism ${ }^{76}$ 


\section{a Tissue damage}
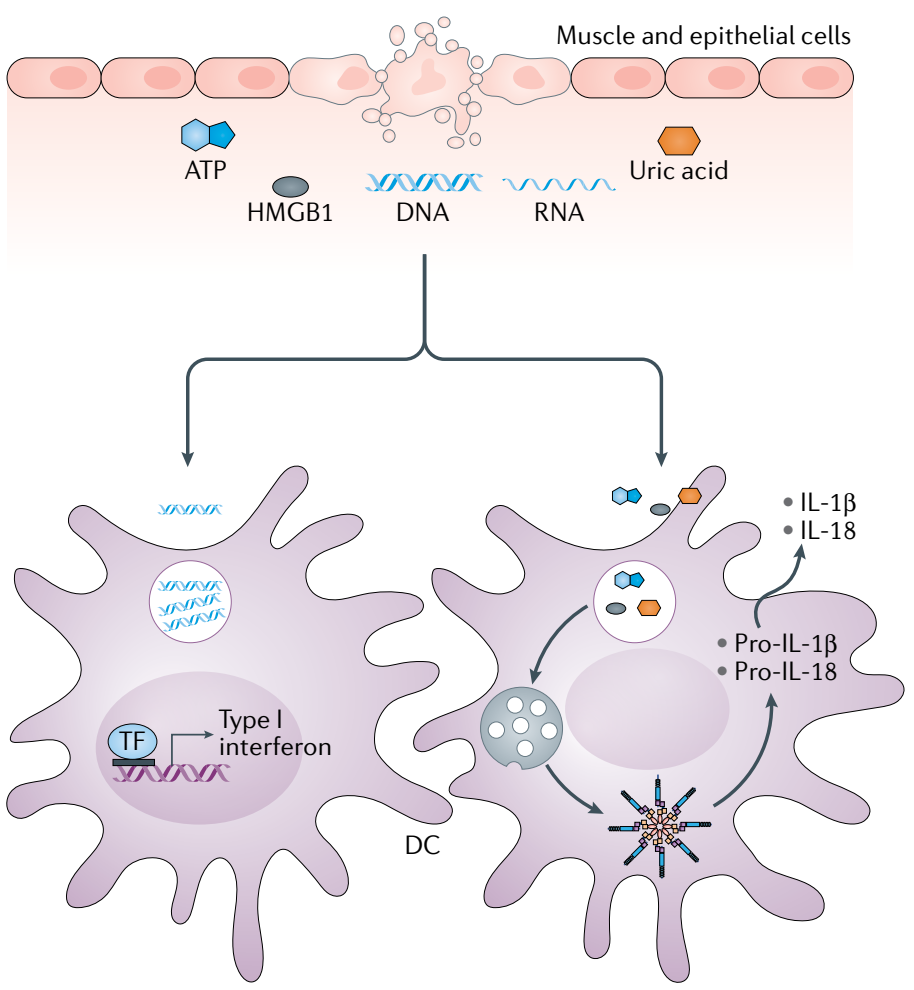

c Metabolic modulation

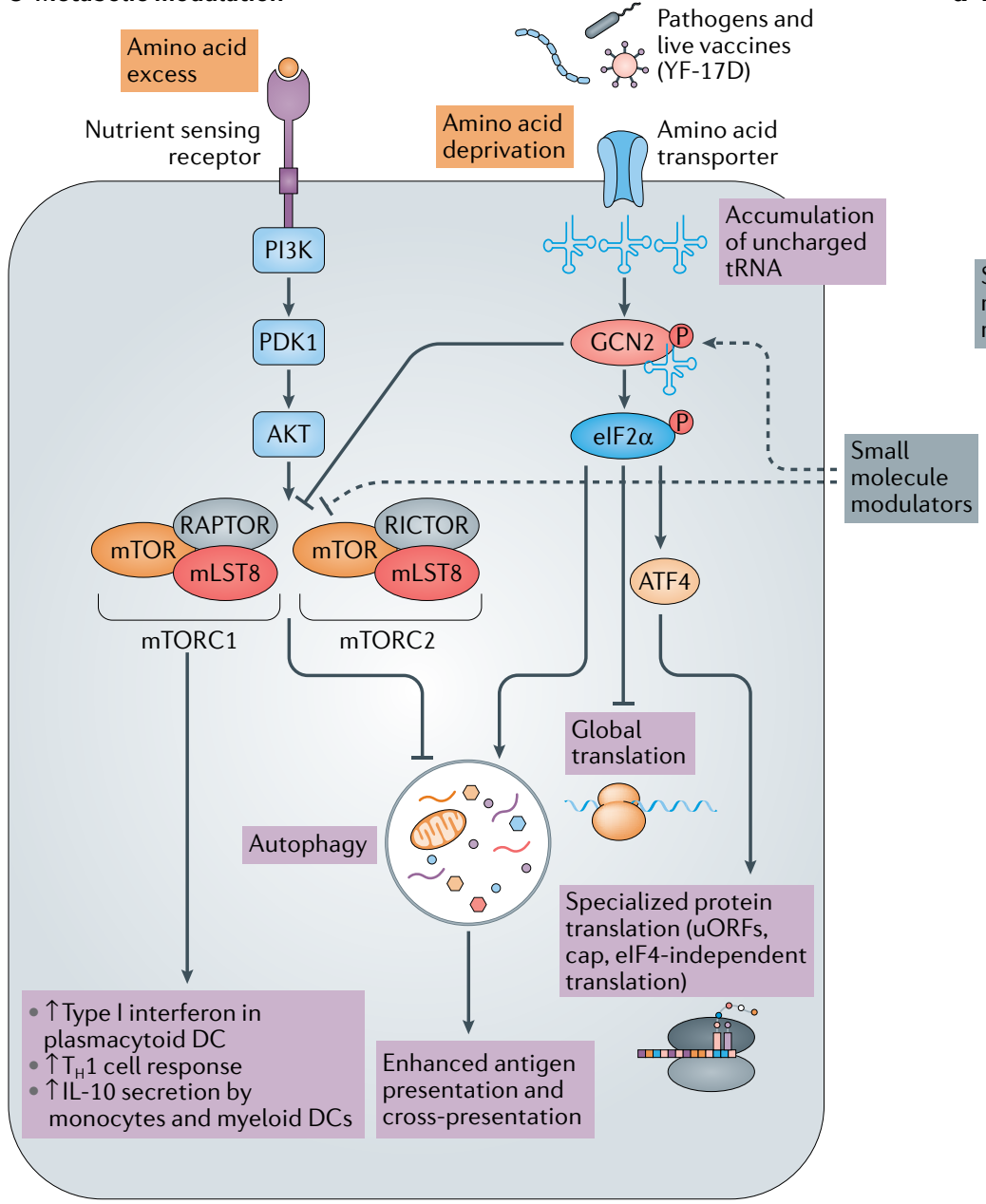

\section{b Cell death}

Small molecules to induce specific cell death pathways

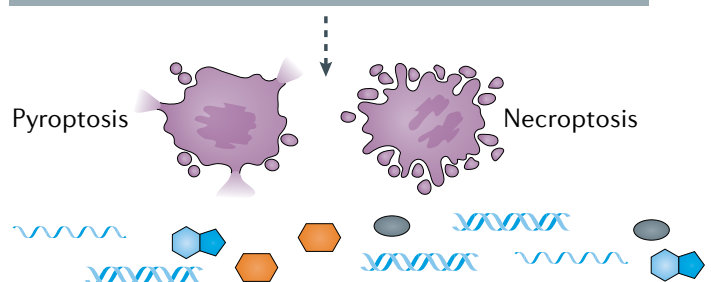

\section{DAMP-mediated
activation}

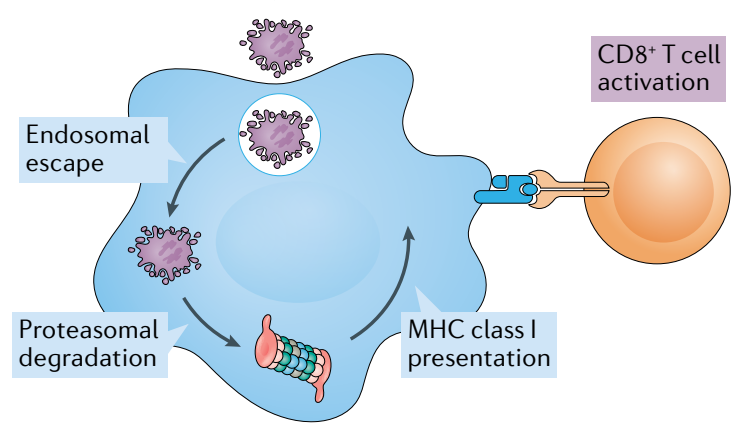

d Epigenetic modulation

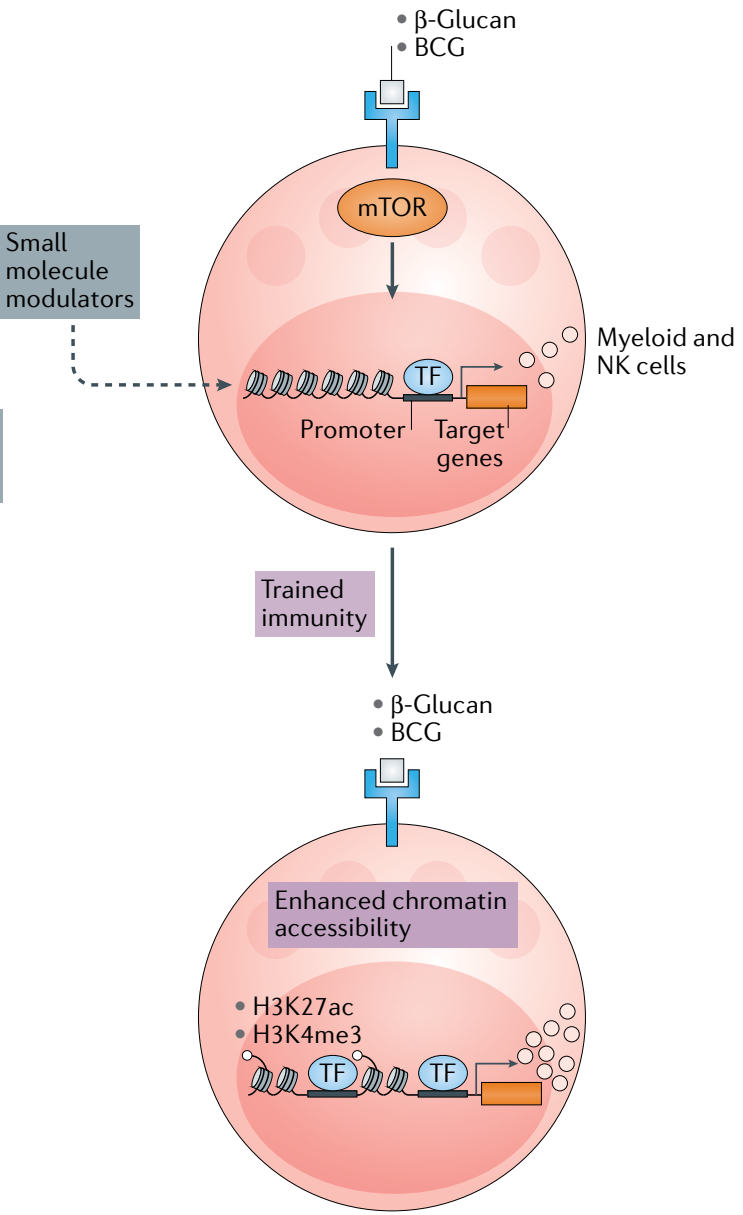


4 Fig. 2 | Beyond pattern recognition receptors. Recent studies have yielded insights into novel pathways that could be targeted for adjuvant activity. a | Tissue damage caused by trauma, infection and autoimmunity results in release of a multitude of damage-associated molecular patterns (DAMPs), including nucleic acids, uric acid, ATP and proteins such as high mobility group box 1 (HMGB1), that activate the innate immune system. $\mathbf{b}$ |Cell death induced by different stimuli also releases DAMPs. Of particular interest is the mechanism of cell death induced by different stimuli. Specialized cell death pathways, such as necroptosis and pyroptosis, can activate innate immune cells. Small molecules that induce specific cell death pathways could be effective adjuvants. c|Cellular metabolism is the third concept that is emerging as a central regulatory network of immune responses. Immune cells, such as dendritic cells (DCs), have a distinct metabolic state in different tissues. The insights stemmed from the systems analysis of yellow fever vaccine-induced immune responses in humans, in which the amino acid sensor $\mathrm{GCN} 2$ emerged as an early correlate of lasting $\mathrm{CD} 8^{+} \mathrm{T}$ cell responses. GCN2 activation in DCs by the yellow fever vaccine enhances antigen presentation to T cells via autophagy. Furthermore, the central metabolic regulator mTOR is shown to have various effects on innate immune responses, especially of DCs. $\mathbf{d}$ |Vaccines such as Bacillus Calmette-Guérin (BCG) and pathogen-associated molecular patterns (PAMPs) such as $\beta$-glucan induce epigenetic changes that maintain the innate immune system at an alarming state for extended periods. Small molecules targeting appropriate cell types offer attractive components of novel adjuvants. $\mathrm{H} 3 \mathrm{~K} 4 \mathrm{me} 3$, histone $\mathrm{H} 3$ trimethylated at Lys4; H3K27ac, histone $\mathrm{H} 3$ acetylated at Lys27; MHC, major histocompatibility complex; NK cell, natural killer cell; $P$, phosphorylation; TF, transcription factor; $\mathrm{T}_{H} 1$ cell, Thelper 1 cell; uORF, upstream open reading frame.

(FIC. 2b). Taken together, these findings suggest that small molecules that can be targeted to macrophages in lymph nodes to transiently induce RIPK3-mediated pathways, including cell death, might represent adjuvants that stimulate $\mathrm{CD}^{+} \mathrm{T}$ cell responses (FIG. 2b).

Metabolic adjuvants. Another concept that has gained momentum in recent years is the notion that the metabolic state of myeloid cells, such as macrophages and DCs, can programme their innate response and capacity to stimulate T cells ${ }^{6,155}$. Thus, DCs at different maturation stages, or in different tissues, have distinct metabolic states ${ }^{155,156}$. For example, the central metabolic regulator mTOR complex has a major role in mediating TLR-induced type I interferon secretion by plasmacytoid DCs ${ }^{157}$, affects IL-12 secretion in myeloid DCs and metabolically reprogrammes lung DCs to skew allergic inflammation from eosinophilic $\mathrm{T}_{\mathrm{H}} 2$ to neutrophilic $\mathrm{T}_{\mathrm{H}} 17$ cell polarity, via a mechanism that results in enhanced IL-23 and fatty acid oxidation ${ }^{156}$.

In addition, the amino acid sensor eIF $2 \alpha$ kinase GCN2 can modulate the innate function of myeloid cells, and their capacity to induce $\mathrm{T}$ cell responses. For example, YF-17D stimulates GCN2 activation in DCs, leading to enhanced autophagy and antigen presentation to $\mathrm{CD}^{+}$and $\mathrm{CD} 4^{+} \mathrm{T}$ cells ${ }^{158}$ (FIG. 2c). By contrast, GCN2 results in suppression of inflammasome activation, via a mechanism involving the sequestration of reactive oxygen species (ROS), an activator of NLRP3, in autophagosomes ${ }^{159}$. Furthermore, GCN2 alters myeloid cell function and promotes immunosuppression against antitumour response through a mechanism involving increased translation of the transcription factor CREB2 (also known as ATF4), which was required for maturation and polarization of macrophages and myeloid-derived suppressor cells ${ }^{160}$. These results suggest that pharmacological modulation of metabolic regulators such as mTOR or GCN2 in DCs may represent adjuvant strategies to simulate immune responses.
Epigenetic adjuvants. Another concept that has emerged recently is that of innate immune memory or 'trained immunity ${ }^{161-163}$. It has been proposed that myeloid cells, such as monocytes and macrophages ${ }^{163}$ or NK cells ${ }^{164}$, acquire memory-like characteristics following stimulation with PAMPs. This 'innate memory' depends on sustained epigenetic programming induced upon a primary stimulus that causes cells to be either hyperresponsive or hyporesponsive following a secondary stimulation ${ }^{161,162}$. Vaccines (such as BCG) and PAMPs (such as $\beta$-glucan) induce histone $\mathrm{H} 3$ trimethylated at Lys4 (H3K4me3) and H3 acetylated at Lys27 (H3K27ac) in monocytes and macrophages (FIG. 2d). These changes were maintained up to several weeks after the elimination of the stimuli, resulting in an enhanced epigenetic status. During a secondary stimulus with the same or a different PAMP, this enhanced epigenetic status induces enhanced gene expression ${ }^{163,165,166}$. An attractive possibility is to target small molecules to the appropriate cell types as pharmacological modulators to reprogramme the epigenetic landscape of innate immune memory ${ }^{167}$. In particular, one could envision small molecules that target monocytes or other myeloid cells to undergo epigenetic reprogramming that stimulates heightened activation of particular facets of the innate response, such as an antiviral state that provides heightened resistance to virus, for some period of time, perhaps a few weeks or so. Such an epigenetic adjuvant could be used to imprint an enhanced antiviral state that confers resistance against a broad array of viruses in humans. The use of such epigenetic adjuvants may be especially advantageous during a pandemic, such as the COVID-19 pandemic, in conferring enhanced antiviral resistance, for a limited period of perhaps a few weeks, in a susceptible population.

\section{Continuing education by adjuvants}

One of the dominant paradigms in immunology is that the rapidly responding innate immune system senses microbial stimuli and then programmes the ensuing adaptive immune response. However, emerging data also point to a potential continuing role for adjuvant signals in later stages of the immune response, by directly targeting cells of the adaptive immune system such as B cells. Immunization with a vaccine results in uptake of antigens by DCs, and their activation by the adjuvants contained in the vaccine, via TLRs or other PRRs ${ }^{6,23}$ (FIG. 3). Activated DCs present antigens to naïve antigen-specific CD4 ${ }^{+} \mathrm{T}$ helper cells, which are found in the $\mathrm{T}$ cell-rich areas of the lymphoid organs. The magnitude and type of the T helper cell response induced depends greatly on the nature of the adjuvant and the PRRs triggered. Activated T helper cells migrate to the interface between the $\mathrm{B}$ cell follicle and the $\mathrm{T}$ cell area, where they stimulate the clonal expansion of antigen-activated B cells. These activated antigen-specific B cells migrate to the medullary cords in the lymph nodes and differentiate into short-lived plasma cells ${ }^{168-170}$. Other activated B cells migrate into $B$ cell follicles to form GCs. In addition, some T helper cells upregulate BCL-6, which induces expression of CXCR5 and CCR7, enabling migration to the T cell-B cell border, where they interact with antigen-specific B 
cells ${ }^{168,170}$. These $\mathrm{T}_{\mathrm{FH}}$ cells provide IL-21 and CD40L signals, which are essential for B cell proliferation and differentiation of GC cells or plasma cells. Antigen-specific $B$ cells compete with each other for $T_{F H}$ cell help for cytokines and co-stimulation signals at the T cell-B cell border based on the amount of peptide MHC class II antigens presented by the $\mathrm{B}$ cells to the $\mathrm{T}_{\mathrm{FH}}$ cells. In turn, signals from B cells, including CD40L, IL-6, IL-12 and IL-23, stimulate the development of $\mathrm{T}_{\mathrm{FH}}$ cells and their migration into the nascent GCs, where they play a central part in orchestrating the development of memory B cells and LLPCs ${ }^{168,170}$. Therefore, $\mathrm{T}_{\mathrm{FH}}$ cells represent attractive targets for adjuvant design; in particular, for a deeper mechanistic understanding of the subsets of DCs and the PPRs on such subsets that need to be triggered to induce enhanced $\mathrm{T}_{\mathrm{FH}}$ cell responses. In this context, our recent work in NHPs has demonstrated that the synthetic TLR7/TLR8 ligand known as 3M-052 formulated in nanoparticles or in alum induces a very high magnitude of $\mathrm{T}_{\mathrm{FH}}$ cell response and GC response, as well as remarkably persistent bone marrow LLPCs that persist at very high levels for 70 weeks, which was the termination of the study ${ }^{35}$. On the basis of these results, 3M-052 formulated in alum is currently under evaluation as a vaccine adjuvant for HIV Env antigens in humans (ClinicalTrials.gov NCT04177355).

Although targeting particular DC subsets via specific PRRs during the early 'innate' phase of the immune response is a well-established mechanism of adjuvant action, emerging evidence suggests that adjuvants can also provide 'continuing education' to the $\mathrm{B}$ cell response even during the GC stage of development. Within GCs, activated antigen-specific $B$ cells proliferate rapidly and undergo somatic hypermutation in the genes encoding their immunoglobulin receptors. As a result, new specificities of B cells are created, including those with a heightened capacity to bind the immunizing antigen ${ }^{169}$. GC B cells compete for antigens that are expressed as immune

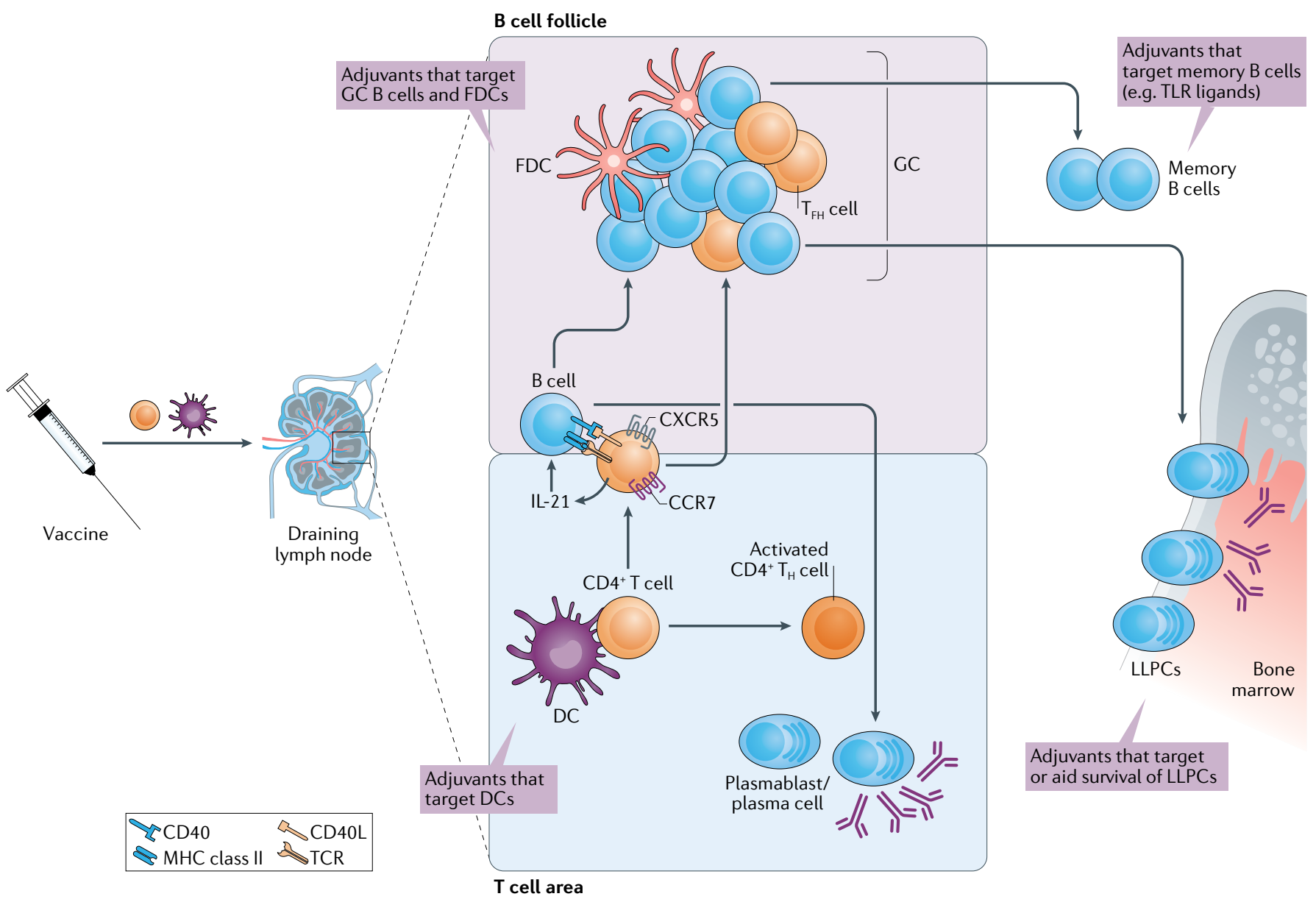

Fig. 3 | Beyond the innate/adaptive paradigm, continuing education by adjuvants. The innate immune system sensing adjuvants and programming the ensuing adaptive immune responses is the current model of how adjuvants function. Activated dendritic cells (DCs) present antigens to naïve antigen-specific $C D 4^{+} T$ helper cells ( $T_{H}$ cells) in $T$ cell areas. Some activated $\mathrm{T}_{\mathrm{H}}$ cells upregulate $\mathrm{CXCR5}$, which mediates their migration to the interface between the B cell follicle and the T cell area, where they express IL-21 and CD40L that stimulate the clonal expansion of antigen-activated B cells. Although some antigen-specific B cells migrate to the medullary cords and differentiate into short-lived plasma cells, other activated B cells migrate into B cell follicles to form germinal centres (GCs). B cells in GCs can subsequently differentiate into memory $B$ cells that recirculate, or long-lived plasma cells (LLPCs) that migrate to the bone marrow. Many adjuvants are known to work primarily by targeting DCs to induce their activation and antigen presentation, but emerging studies demonstrate that adjuvants such as Toll-like receptor (TLR) ligands can also target B cells. Therefore, potentially novel adjuvant targets could include B cell subsets in GCs, bone marrow LLPCs and other cell types that aid survival of LLPCs, follicular DCs (FDCs) and T follicular helper cells ( $\mathrm{T}_{\mathrm{FH}}$ cells). MHC, major histocompatibility complex; TCR, T cell receptor. 
complexes on follicular dendritic cells (FDCs), and those with the highest affinity for antigens are preferentially selected and differentiate into memory B cells or LLPCs that migrate to the bone marrow ${ }^{169}$. This process results in 'affinity maturation' of the antibody response. Activated B cells express TLRs and can respond to TLR stimulation $^{33,171}$. Combination of specific TLRs can drive persistent GC responses ${ }^{33}$ and, as mentioned above, $\mathrm{B}$ cell-intrinsic MyD88 signalling is essential to stimulate antigen-specific B cell and antibody responses ${ }^{33,133}$. In addition, FDCs express TLRs and TLR-mediated activation of FDCs is crucial for GC differentiation and affinity maturation ${ }^{172,173}$. Hence, this mechanism may be key in inducing high-affinity antibodies and the differentiation of LLPCs, which is essential to inducing sustained antibody responses, a major challenge in vaccinology. These observations suggest that adjuvants might not only target DCs during the early phase of the innate response but could also have a continuing role in impacting GC responses and memory $\mathrm{B}$ cell differentiation.

\section{Immunogenicity is context-dependent}

The 'rules of immunogenicity', or how the immune system responds to a given adjuvant or vaccine, depend greatly on the context. For example, the requirements for stimulating naïve $\mathrm{T}$ or B lymphocytes are generally more stringent than those for stimulating memory lymphocytes, and thus adjuvants may be especially needed to stimulate a naïve response $\mathrm{e}^{174-176}$. This issue is important because vaccines against many infections such as influenza often need to stimulate recall responses, because most people have been exposed to influenza. However, sometimes a vaccine (such as a vaccine against pandemic influenza) may need to evoke responses against new antigenic epitopes, in the face of clonal competition from memory B cell clones that recognize conserved epitopes. This concept has been termed the 'original antigenic $\sin ^{177}$ and proposed to be a reason for lower vaccine efficacy observed during certain seasons ${ }^{178,179}$. Adjuvants that selectively activate naïve B cells or APC subsets that stimulate naïve $B$ cells may offer potential to overcome the challenges of the original antigenic sin. Adjuvants such as AS03 can overcome this; a recent study shows that vaccination of humans with the avian influenza $\mathrm{H} 5 \mathrm{~N} 1$ vaccine adjuvanted with AS03 results in enhanced stimulation of naïve B cells specific to $\mathrm{H} 5 \mathrm{~N} 1$, relative to the stimulation induced by a non-adjuvanted H5N1 vaccine ${ }^{180}$.

Another example of context-dependency is that the immunological states of individuals within a given population, or those in geographically distinct populations, can vary because of differences in genes, the environment or the microbiome ${ }^{181-183}$. Within a given population, individuals at the extremes of age can have different or suboptimal immune responses to vaccination. Indeed, the efficacy of unadjuvanted influenza vaccines in older people is consistently poor ${ }^{184,185}$, and adjuvanted vaccines increase the potency and effectiveness of influenza vaccines in this population ${ }^{186,187}$. Furthermore, the CpG 1018-adjuvanted hepatitis B vaccine Heplisav-B is much more effective in older adults than the alum-adjuvanted HBV vaccine, using a very similar antigen ${ }^{188}$. Similarly, the AS01-adjuvanted recombinant varicella zoster vaccine has a remarkably high efficacy of $97.2 \%$ in populations aged older than 50 years (REF. ${ }^{93}$ ). At the other extreme of age, the novel TLR7/TLR8 adjuvant 3M-052 overcomes hyporesponsiveness of the newborn immune system in macaques, to elicit more potent immune responses $^{189}$. However, mechanistic insights into the innate immune system and the way it responds to different stimuli in young and older people are scarce ${ }^{190,191}$.

There can also be differences in vaccine-induced immunity in populations living in different geographical regions of the world. For example, it is well known that vaccines against polio, rotavirus, malaria and yellow fever provide less protection for populations in Africa and Asia, relative to those in Europe or the USA ${ }^{181}$. The microbiome is emerging as a central player in the human immune system, and it is suggested that differences in the composition of the microbiome in distinct populations could, in part, account for these differences ${ }^{181,192-194}$. Indeed, a recent study suggests that ablation of the microbiota in humans with broad-spectrum antibiotics results in a marked reduction in H1N1-specific binding antibody responses and neutralization titres to seasonal influenza vaccination in humans ${ }^{194}$. Strikingly, this effect was only observed in subjects who had relatively low baseline titres against influenza, and who had not been vaccinated against influenza or acquired influenza in the 3 years preceding their enrolment into the study ${ }^{194}$. This suggests that the microbiota had provided some endogenous adjuvant signal in the context of influenza vaccination. In addition to this effect on the adaptive immune system, antibiotic administration was observed to stimulate a profound alteration in the metabolome in the serum, with a 1,000-fold reduction in secondary bile acids, which was highly correlated with enhanced inflammation, notably signatures of inflammasome activation ${ }^{194}$. These results reveal a key role for the microbiome in modulating vaccine responses and raise the concept of the microbiota as an endogenous vaccine adjuvant. A practical example of this is the use of probiotics in vaccination and immunotherapy ${ }^{195}$. However, although there are many efforts at evaluating the effect of probiotics on the immune response to vaccination, the results have been variable because of the relatively small study sizes. Therefore, future research should be aimed at devising novel adjuvants, such as small molecules, that harness the microbiome-host axis (for example, metabolites such as secondary bile acids) to regulate immunity to vaccination. Furthermore, it will be important to investigate the mechanisms of the rules of immunogenicity in different populations, such that these can be harnessed in designing adjuvants that are optimally suited to such populations.

\section{The power of adjuvant formulation}

Synthetic molecules versus natural products. Although the most successful adjuvants available right now, those included in licensed products, consist mainly of components derived from natural sources, we would not suggest that this necessarily reflects the best path to 'success' in the early twenty-first century. It must be acknowledged that the components used in the most successful adjuvants were generally available in the early 1990s and 
before. Hence, they were the best materials to include in product development plans back then, as they were readily available and potent, even if their mechanism of action was often poorly understood. However, now that there is greater appreciation of the critical role of innate immunity on adjuvant action, synthetic chemistry facilitates the development of novel molecules, which can be better designed for the purpose, as pure agonists. Such an approach led to the discovery of small molecule immune potentiators (SMIPs), which are currently in various stages of preclinical and clinical evaluation as adjuvants ${ }^{196-199}$. Although the initial programmes focused on the discovery of TLR agonists, the search for novel agonists of other innate activation systems soon followed ${ }^{200-202}$. The inherent characteristics of these newly discovered adjuvants make them more suitable for product development than the original natural molecules that they will likely come to replace. Ideally, the newly discovered adjuvants are small molecules that can be designed as pure agonists, with mechanistic insights allowing modulation of the level of agonism to control activation signals.

Established formulation versus creating a novel formulation approach. Because such small molecule agonists benefit greatly from particulate presentation, chemical manipulation can render them suitable to be formulated into preferred delivery systems, with flexibility in solubility and compatibility profiles ${ }^{203-205}$. Recent research in animal models has highlighted the value of nanoparticle-based vaccines. Here, the physical properties of nanoparticles, such as their size and the antigen density on their surface, can influence their immunogenicity ${ }^{204,205}$. In addition, glycosylated antigens displayed in a multimeric form on the surface of nanoparticles can engage with innate immune defence proteins such as mannose binding lectin (MBL), which facilitates their rapid shuttling to FDCs in GCs, leading to enhanced antibody responses ${ }^{206}$. Experiments in mice and macaques indicate that formulations can also modulate the release kinetics of antigens, which can affect the magnitude of antibody responses ${ }^{38,207}$.

There are several formulation approaches already established that are used for the adjuvants included in licensed products. These include insoluble aluminium salts used as an adsorbent, a low oil content that is easy to inject, oil-in-water emulsions and liposomal delivery systems. Although these systems are very different compositionally, they share some significant similarities. They are all fabricated as nanoparticles, although insoluble alum adjuvants are typically stable aggregates of nanoparticles, which aggregate into particulates of a mean size of several microns. Importantly, alum, emulsions and liposomes can also function as effective 'delivery systems' for both antigens and immune potentiators, including TLR agonists (most notably TLR4, TLR7 and TLR9). 3M-052 has also been formulated using several different approaches, including adsorption to alum and encapsulation in liposomes ${ }^{208,209}$.

Finally, a major challenge in the development of these delivery systems is translation to humans. Controlled release of antigen through polymeric particles has always been attractive conceptually and has gained much support as there were already well-established biodegradable polymers available for use in humans. However, many challenges emerged in the early years of this approach, which were not able to be overcome despite much effort; these challenges have been summarized previously ${ }^{210}$. In brief, most recombinant antigens were degraded by the nanoparticle encapsulation process or were released only partially after encapsulation, and entrapment was often inefficient, resulting in high cost due to 'wasted' antigen. Although the in vivo performance in small animals was often good, there was no clear path to product development owing to several inherent liabilities. These include the fact that the particles typically required expensive aseptic manufacturing in very specialized facilities, and that the release and quality control assays were very challenging, as the entrapped antigen needed to be recovered from the particle to be assayed and quantified. Hence, a key lesson is that there are many reasons why a technology may not be viable beyond small-scale studies in research laboratories or may not be suitable for rational vaccine development.

A recent example of the success of translating a concept to humans is the ongoing clinical programme that followed on from the initial SMIP discovery ${ }^{211}$. After the identification of novel TLR7 agonists, although there were preclinical data showing the possibility of using multiple delivery systems, the decision taken was to clinically evaluate the molecule adsorbed to alum. The reasoning was that alum is well established, safe and effective, and is already well known to regulators. Hence, the new molecule is initially presented in a 'familiar' format, so all questions can be focused on the clinical profile of the new compound only, as it can easily be evaluated in conjunction with established safe and effective vaccines $^{211}$. The manufacturing approach was also established for alum, and is used in many products and allows the potential of the optimal product presentation form, as liquid single vials or pre-filled syringes. Moreover, this approach had already been established as a practical way to deliver TLR agonists, owing to the success of AS04 $\left(\right.$ REF. $\left.^{78}\right)$. Hence, a previously successful path to product development was followed for a vaccine incorporating a novel adjuvant. Importantly, the flexibility inherent in the SMIP approach allowed chemical modification of the molecule to ensure adsorption to alum adjuvants, allowing rapid clinical evaluation, without the need to establish a new manufacturing process. Encouragingly, so far, the TLR7 agonist adsorbed to alum looks potentially safe and well tolerated in humans $\mathrm{s}^{212}$.

Including only what is necessary. Ensuring that each component is necessary and adds clear value, while not introducing significant unjustified liabilities, enables the rational building of improved adjuvants. AS01 is currently the most successful adjuvant included in a licensed product, based on the level of efficacy achieved in the product, with efficacy $>97 \%$ against varicella zoster ${ }^{93}$. Prior to the development of AS01, the key components, MPL and QS-21, were both already established as adjuvants and had been explored independently for quite some time. However, an insight that was key to the development of 
the AS01 adjuvant was that these two molecules together resulted in a synergy of innate activation ${ }^{213}$. The two molecules together induced an innate activation pattern that could not be induced by either one alone, regardless of the dose level. Importantly, to enable this synergy, a liposomal delivery system was developed, which allowed the co-delivery of the two immune potentiators to the same immune cell populations. This co-delivery could not be accomplished with the established alum or emulsion approaches, and hence the development of liposomes was fully justified. Importantly, the liposomal formulation was also effective in reducing the potential of QS-21 to induce significant local and systemic reactogenicity. Hence, AS01 stands as an excellent example of how strong justifications are needed to add additional components but new approaches can be justified based on outcomes. However, as described below, novel system vaccinology approaches could be used to identify novel adjuvant pathways and formulations that could be harnessed to design the next generation of adjuvants.

\section{A new framework}

\section{Systems vaccinology}

The established approach to the development of vaccines containing novel adjuvants has been described as one of the slowest processes in medicine ${ }^{1}$. For decades, adjuvant development has relied on systematic testing of candidate molecules in mice, advancement of promising candidates into NHP models and eventual testing in humans (FIG. 4a). Yet, out of all the adjuvants that have shown great immunogenicity and efficacy in animal models, only a handful have proven to be safe and effective in humans. A major reason for this lack of translation from mice to humans includes their evolutionarily divergence some 62 million years ago, along with the resulting important immunological differences (such as differences in TLR7 expression in DC subsets) despite the broad similarities of their immune systems (both mice and humans have $\mathrm{T}$ and $\mathrm{B}$ lymphocytes ${ }^{183}$. These main differences underscore the need to harness a human model in the testing of adjuvants. Recent advances in systems vaccinology have transformed our ability to probe the immune response to vaccination in humans, with an unprecedented degree of precision $^{22,25,214-216}$.

For decades, vaccine manufacturers have relied on a single measure, typically serum antibody titres, to assess immune responses to vaccination. A major limitation of this approach is that it does not capture the complexity of the immune response to vaccination, and therefore may fail to identify correlates and critical mechanisms of protective immunity. Systems vaccinology uses high-throughput technologies such as transcriptomics, metabolomics, high-dimensional cytometry and epigenomics to comprehensively analyse the immune responses to vaccination and to use the data generated through such analyses to delineate correlates and mechanisms of vaccine immunity ${ }^{22,183}$. The first studies to use systems-based approaches to study vaccine responses identified transcriptional signatures induced within 1-7 days of vaccination that correlated with the magnitude of the ensuing antigen-specific $\mathrm{CD} 8^{+} \mathrm{T}$ cell response and neutralizing antibody response that was measured
60-90 days later ${ }^{25}$. This study revealed that the yellow fever vaccine triggered multiple TLRs, as well as RIG-I and MDA5, and identified innate signatures that predict the immunogenicity of the vaccine. Further analysis using machine learning approaches demonstrated the capacity of these signatures to predict the magnitude of the ensuing antigen-specific $\mathrm{CD} 8^{+} \mathrm{T}$ cell response and neutralizing antibody response in an independent study of subjects who received the yellow fever vaccine ${ }^{25}$. This study provided a proof of concept that systems biological approaches could be used to predict vaccine immunity. In an independent study, Gaucher et al. identified similar signatures induced by the yellow fever vaccine that correlated with the ensuing adaptive immune response ${ }^{215}$. Following these studies, several groups have used this approach to investigate immune responses to vaccination against several other diseases including influenza ${ }^{216-221}$, malaria ${ }^{22-224}$, meningococcal and pneumococcal infections ${ }^{225,226}$ and varicella zoster virus ${ }^{227}$. Importantly, systems vaccinology approaches have been used to identify common vaccine-induced signatures across multiple influenza seasons and in diverse populations (older people, people with diabetes $)^{217}$. Such studies have identified signatures that correlate with the durability of the antibody response $\mathrm{e}^{217}$.

Systems vaccinology studies have yielded many novel mechanistic insights about vaccine response. For example, expression of TLR5 was shown to be induced within a few days of vaccination and correlated strongly with the antibody response several weeks later ${ }^{216}$. Subsequent experiments with mice revealed that antibody responses to vaccination with the seasonal influenza vaccine were impaired in mice deficient in TLR5. This might be due to flagellin from the intestinal microbiota signalling through TLR5 and providing an adjuvant signal to enhance the antibody response. Thus, vaccination of mice treated with broad-spectrum antibiotics or germ-free mice resulted in impaired antibody responses to influenza vaccination $^{228}$. On the basis of these studies, we performed a study in humans to assess the impact of the microbiota on immune responses to the seasonal influenza vaccine, by administering broad-spectrum antibiotics to healthy humans before and after seasonal influenza vaccination ${ }^{194}$. The results revealed that in subjects with low pre-existing antibody titres, microbiome loss resulted in significant impairment in the H1N1-specific neutralization and binding IgG1 and IgA antibody responses. In addition, there was an enhanced inflammatory response (including signatures of inflammasome activation) and a 1,000-fold reduction in secondary bile acids, which was highly correlated with the inflammatory signature ${ }^{194}$. Multi-omics integrative analysis revealed significant associations between bacterial species and metabolic phenotypes, highlighting a key role for the microbiome in human immunity. These studies reveal the power of systems vaccinology approaches: first, in identifying molecular predictors of the vaccine response in humans; then, in experimentally validating them in mouse models; and, finally, in testing these mechanistic insights in a new human study. This highlights the seamless continuum of human immunology studies and mechanistic studies in mice. Additional mechanistic insights that resulted 
a Current model

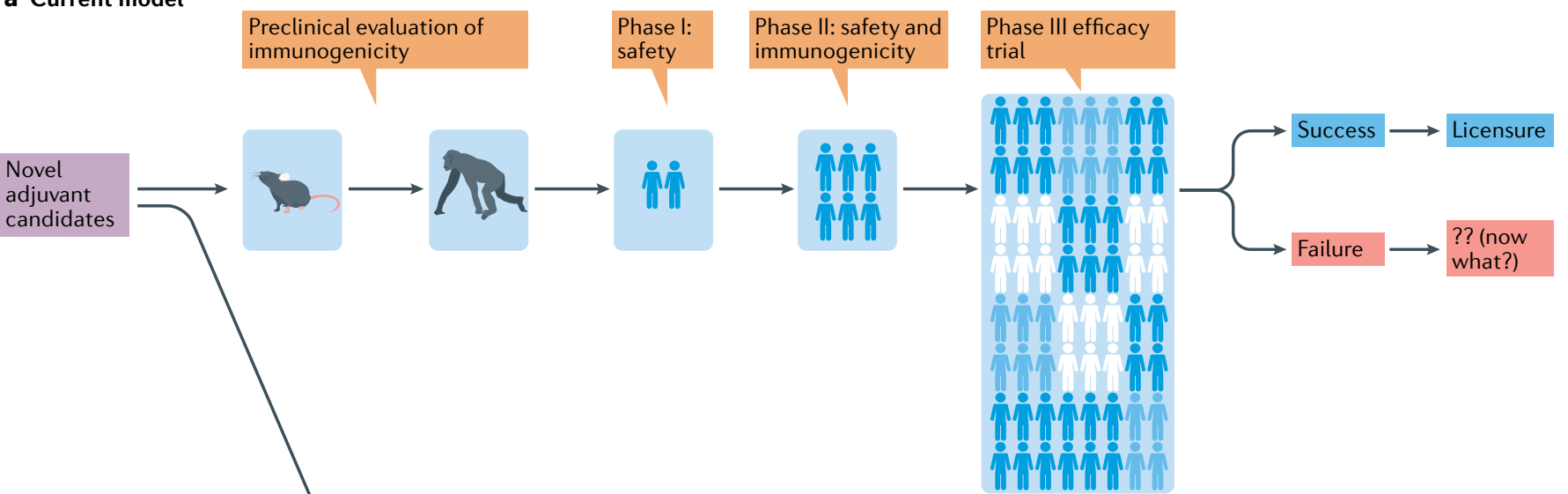

b New model

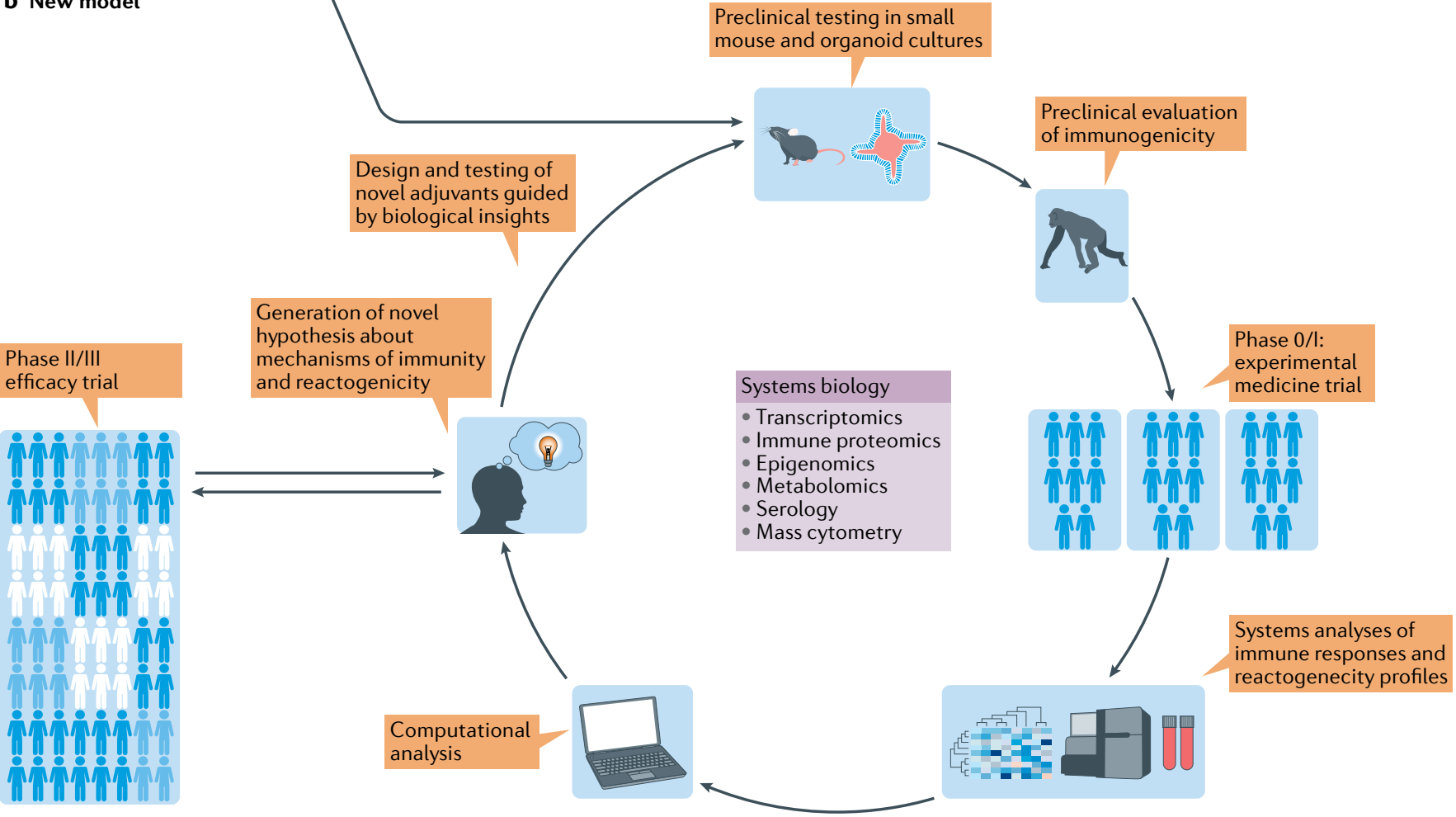

Fig. 4 | A new framework for development of adjuvants. a | The current model of developing vaccines containing novel adjuvants represents a linear progression from the systematic testing of novel candidates in mice, to the advancement of promising candidates to testing in non-human primates (NHPs), and the eventual testing in humans in multiple phases of clinical trials. $\mathbf{b}$ | The new model we propose relies on a process of iterative testing in mice, organoid cultures, NHPs and humans. We advise the early use of small-scale experimental human trials and the use of systems biology approaches to generate multiparametric immunological read-outs, which enable the generation of novel hypothesis and adjuvant concepts that can be retested in preclinical models.

from systems vaccinology studies include demonstration of the role of the amino acid sensing molecule GCN2 in regulating DC function to stimulate $\mathrm{T}$ cell responses to the yellow fever vaccine ${ }^{158,159}$.

\section{Advancing adjuvant development}

A new framework for adjuvant development could be one that places greater emphasis on testing many potential adjuvant concepts in small clinical trials (phase 0/I), early in their developmental pipeline (BOX 3). Thus, novel adjuvants can be rapidly tested in small phase I (phase 0 ) human trials and systems vaccinology approaches used to obtain mechanistic insights. For example, Sékaly and colleagues studied the innate immune response in humans to synthetic double-stranded RNA (polyinosinic:polycytidylic acid (poly(I:C)) stabilized with poly-L-lysine (poly(ICLC))), an agonist for TLR3 and MDA5 (REF. ${ }^{229}$ ). Transcriptional analysis of blood samples, 


\section{Box 3 How systems vaccinology can advance new adjuvants}

\section{Discovering the mechanisms of action of established adjuvants to discover new adjuvants. There is a paucity of detailed understanding about the molecular and cellular mechanisms of action of adjuvants that are currently used in licensed vaccines, including squalene-based adjuvants such as MF59 and AS03 as well saponin-based adjuvants such as AS01b. Systems vaccinology approaches to probe the molecular networks induced by such adjuvants in humans can illuminate potential mechanisms of action, which can then be experimentally validated in animal models ${ }^{23,182}$. Such a detailed mechanistic understanding can then help design novel adjuvants.}

\section{- Early phase 0/I testing in humans to accelerate the adjuvant development process.} For decades, adjuvant testing has progressed in a linear, unidirectional path, starting with preclinical testing in mice, followed by testing the most promising candidates in non-human primates and progressing slowly to clinical testing in humans. However, translation of promising candidate adjuvants into humans has been frustrated by differences in immunogenicity in mice and humans. Furthermore, knowledge of the failure in translation typically occurs very late in the developmental process, typically years after preclinical studies. Therefore, testing candidate adjuvants in humans earlier in the developmental process, in small phase $0 / \mathrm{l}$ trials, and using systems-based approaches to define signatures of immunogenicity will help accelerate adjuvant development ${ }^{229,238}$. This will also help benchmark adjuvant and formulation signatures with other successful vaccines ${ }^{30,218,225}$

- Identifying mechanisms and correlates of reactogenicity. A major consideration in adjuvant development is safety. Sometimes, adjuvants can induce undesirable reactogenicity, which typically occurs within a few days of vaccination, and can be local (such as erythema, swelling or pain) or systemic (such as fever, myalgia, headache and flu-like symptoms). In addition to such reactogenicity, in rare cases, adverse events such as anaphylactic reactions after administration of an adjuvanted vaccine can also occur ${ }^{239}$. Retrospective, nested, case-control studies can be used to analyse samples from such adverse events compared with controls to identify mechanisms underlying the host response to adverse events.

after subcutaneous administration of poly(ICLC), showed upregulation of genes involved in multiple innate immune pathways in all subjects, interferon and inflammasome signalling, similar to the signature observed with the yellow fever vaccine. Comparative transcriptional analysis showed that several innate immune pathways were similarly induced in volunteers immunized with the highly efficacious yellow fever vaccine ${ }^{229}$. Therefore, a chemically defined PRR agonist such as poly(ICLC) can be a reliable and authentic microbial mimic for inducing innate immune responses in humans. Similar studies can be done in humans vaccinated with novel adjuvants that can be 'benchmarked' against successful vaccines. In addition, systems vaccinology studies can provide insights into the mechanisms of action of adjuvants in humans. This is of value even for adjuvants that are currently used in licensed vaccines, including squalene-based adjuvants, such as AS03 and MF59, and saponin-based adjuvants, such as AS01b, which do not seem to activate the canonical TLR-dependent or other PRR-dependent pathways of innate activation. Regarding saponin-based adjuvants, the results from a recent clinical trial for a COVID-19 vaccine with another saponin-based adjuvant known as Matrix-M1 (BOX 2) demonstrate a high magnitude of neutralizing antibody titres ${ }^{230}$, but there is a lack of detailed knowledge about the molecular and cellular mechanisms by which such saponin-based adjuvants mediate their effects ${ }^{231}$. Furthermore, systems vaccinology approaches could be used to define not only mechanisms of action of adjuvants but also the underlying mechanisms by which formulations work, the underlying mechanisms of adverse reactions that occur soon after vaccination and the rational design of optimal formulations for vaccine deliver $\mathrm{y}^{30}$. The results obtained from such phase $0 / \mathrm{I}$ studies will enable the formulation of mechanistic hypothesis about adjuvants that can then be tested in animal models or in vitro human organoid cultures ${ }^{232}$ (FIG. 4b).

Here, we propose an improved new model for rational design and iterative testing of novel adjuvants - an interdisciplinary approach based on systems vaccinology to accelerate adjuvant discovery and development in the clinic (FIG. 4). Based on our experiences in managing new adjuvants through to commercial approval in vaccine products, we would like to highlight the value of the early use of human studies. The 'omics' data generated from such studies can catalyse the formulation of novel hypotheses about the mechanisms by which the candidate adjuvants stimulate robust and durable antigen-specific T and B cell responses. Such hypotheses can then be retested in animal models, and the mechanistic insights that ensue can then be used to design novel adjuvant concepts. Such an approach that harnesses the human model combined with the field of systems vaccinology at an early stage of the adjuvant development pipeline has the transformative potential required to revitalize the science of adjuvants.

Published online 6 April 2021
1. De Gregorio, E., Tritto, E. \& Rappuoli, R. Alum adjuvanticity: unraveling a century old mystery. Eur. J. Immunol. 38, 2068-2071 (2008).

2. Beutler, B. Inferences, questions and possibilities in Toll-like receptor signalling. Nature 430, 257-263 (2004).

3. Kawai, T. \& Akira, S. The role of pattern-recognition receptors in innate immunity: update on Toll-like receptors. Nat. Immunol. 11, 373-384 (2010).

4. Medzhitov, R. Toll-like receptors and innate immunity. Nat. Rev. Immunol. 1, 135-145 (2001).

Nat. Rev. Immunol. 1, 135-145 (2001).
Coffman, R. L., Sher, A. \& Seder, R. A. Vaccine adjuvants: putting innate immunity to work. Immunity 33, 492-503 (2010).

6. Pulendran, B. The varieties of immunological experience: of pathogens, stress, and dendritic cells. Annu. Rev. Immunol. 33, 563-606 (2015) Reed, S. G., Orr, M. T. \& Fox, C. B. Key roles of adjuvants in modern vaccines. Nat. Med. 19, 1597-1608 (2013). Steinman, R. M. \& Banchereau, J. Taking dendritic cells into medicine. Nature 449, 419-426 (2007). Janeway, C. A. Jr. Approaching the asymptote? Evolution and revolution in immunology. Cold Spring Harb. Symp. Quant. Biol. 54, 1-13 (1989).
10. Chow, K. T., Gale, M. Jr. \& Loo, Y. M. RIG-I and other RNA sensors in antiviral immunity. Annu. Rev. Immunol. 36, 667-694 (2018).

11. Hu, M. M. \& Shu, H. B. Innate immune response to cytoplasmic DNA: mechanisms and diseases. Annu. Rev. Immunol. 38, 79-98 (2020).

12. Brown, G. D., Willment, J. A. \& Whitehead, L. C-type lectins in immunity and homeostasis. Nat. Rev. Immunol. 18, 374-389 (2018).

13. Christgen, S. \& Kanneganti, T. D. Inflammasomes and the fine line between defense and disease. Curr. Opin. Immunol. 62, 39-44 (2019).

14. Mangan, M. S. J. et al. Targeting the NLRP3 inflammasome in inflammatory diseases. Nat. Rev. Drug Discov. 17, 588-606 (2018)

15. Kwissa, M., Kasturi, S. P. \& Pulendran, B. The science of adjuvants. Expert Rev. Vaccines 6, 673-684 (2007).

16. Gong, T., Liu, L., Jiang, W. \& Zhou, R. DAMP-sensing receptors in sterile inflammation and inflammatory diseases. Nat. Rev. Immunol. 20, 95-112 (2020).

17. Zindel, J. $\&$ Kubes, P. DAMPs, PAMPs, and LAMPs in immunity and sterile inflammation. Annu. Rev. Pathol. $15,493-518$ (2020)
18. Peltzer, N. \& Walczak, H. Cell death and inflammation - a vital but dangerous liaison. Trends Immunol. 40, 387-402 (2019).

19. Yatim, N., Cullen, S. \& Albert, M. L. Dying cells actively regulate adaptive immune responses. Nat. Rev. Immunol. 17, 262-275 (2017).

20. Janssens, S., Pulendran, B. \& Lambrecht, B. N Emerging functions of the unfolded protein response in immunity. Nat. Immunol. 15, 910-919 (2014).

21. Matzinger, P. Tolerance, danger, and the extended family. Annu. Rev. Immunol. 12, 991-1045 (1994).

22. Pulendran, B., Li, S. \& Nakaya, H. I. Systems vaccinology Immunity 33, 516-529 (2010)

23. Pulendran, B. \& Ahmed, R. Immunological mechanisms of vaccination. Nat. Immunol. 12, 509-517 (2011).

24. Querec, T. et al. Yellow fever vaccine YF-17D activates multiple dendritic cell subsets via TLR2, 7, 8, and 9 to stimulate polyvalent immunity. J. Exp. Med. 203, 413-424 (2006).

25. Querec, T. D. et al. Systems biology approach predicts immunogenicity of the yellow fever 
vaccine in humans. Nat. Immunol. 10, 116-125 (2009).

This study is the first using systems biological approaches to define predictors of YF-17D immunogenicity.

26. Tsuji, S. et al. Maturation of human dendritic cells by cell wall skeleton of Mycobacterium bovis Bacillus Calmette-Guérin: involvement of Toll-like receptors. Infect. Immun. 68, 6883-6890 (2000).

27. Moliva, J. I., Turner, J. \& Torrelles, J. B. Immune responses to Bacillus Calmette-Guérin vaccination: why do they fail to protect against Mycobacterium tuberculosis? Front. Immunol. 8, 407 (2017).

28. Koyama, S. et al. Differential role of TLR- and RLR-signaling in the immune responses to influenza A virus infection and vaccination. J. Immunol. 179, 4711-4720 (2007)

29. Francica, J. R. et al. Innate transcriptional effects by adjuvants on the magnitude, quality, and durability of HIV envelope responses in NHPs. Blood Adv. 1 2329-2342 (2017)

This study compares immunogenicity induced by eight different combinations of adjuvants.

30. Petitdemange, $C$. et al. Vaccine induction of antibodie and tissue-resident $\mathrm{CD} 8^{+} \mathrm{T}$ cells enhances protection against mucosal SHIV-infection in young macaques. JCl Insight 4, e 126047 (2019).

31. Crotty, S. et al. Cutting edge: long-term B cell memory in humans after smallpox vaccination. J. Immunol. 171, 4969-4973 (2003).

32. Hammarlund, E. et al. Duration of antiviral immunity after smallpox vaccination. Nat. Med. 9, 1131-1137 (2003).

This study demonstrates the durability of immune responses elicited by smallpox vaccination.

33. Kasturi, S. P. et al. Programming the magnitude and persistence of antibody responses with innate immunity. Nature 470, 543-547 (2011).

34. Kasturi, S. P. et al. Adjuvanting a simian immunodeficiency virus vaccine with Toll-like receptor ligands encapsulated in nanoparticles induces persistent antibody responses and enhanced protection in TRIM5 restrictive macaques. J. Virol. 91, e01844-16 (2017).

35. Kasturi, S. P. et al. 3M-052, a synthetic TLR-7/8 agonist, induces durable HIV-1 envelope-specific plasma cells and humoral immunity in nonhuman primates. Sci. Immunol. 5, eabb1025 (2020).

36. Slifka, M. K. \& Ahmed, R. Long-lived plasma cells a mechanism for maintaining persistent antibody production. Curr. Opin. Immunol. 10, 252-258 (1998)

37. Slifka, M. K., Antia, R., Whitmire, J. K. \& Ahmed, R. Humoral immunity due to long-lived plasma cells. Immunity 8, 363-372 (1998). This study is the first to demonstrate that LLPCs maintain antibody responses.

38. Cirelli, K. M. et al. Slow delivery immunization enhances HIV neutralizing antibody and germinal center responses via modulation of immunodominance. Cell 177, 1153-1171.e28 (2019).

39. Tam, H. H. et al. Sustained antigen availability during germinal center initiation enhances antibody responses to vaccination. Proc. Natl Acad. Sci. USA 113, E6639-E6648 (2016).

40. Smirnov, D., Schmidt, J. J., Capecchi, J. T. \& Wightman, P. D. Vaccine adjuvant activity of 3M-052: an imidazoquinoline designed for local activity without systemic cytokine induction. Vaccine 29, 5434-5442 (2011)

41. Burny, W. et al. Different adjuvants induce common innate pathways that are associated with enhanced adaptive responses against a model antigen in humans. Front. Immunol. 8, 943 (2017).

42. Nardin, E. H. et al. Phase I testing of a malaria vaccine composed of hepatitis B virus core particles expressing Plasmodium falciparum circumsporozoite epitopes. Infect. Immun. 72, 6519-6527 (2004)

43. Salmon-Ceron, D. et al. Safety and immunogenicity of a recombinant HIV type 1 glycoprotein 160 boosted by a V3 synthetic peptide in HIV-negative volunteers. AIDS Res. Hum. Retroviruses 11, 1479-1486 (1995)

44. Brewer, J. M. et al. Aluminium hydroxide adjuvant initiates strong antigen-specific $\mathrm{T}_{\mathrm{H}} 2$ responses in the absence of IL-4- or IL-13-mediated signaling. J. Immunol. 163, 6448-6454 (1999).

45. Brewer, J. M., Conacher, M., Satoskar, A., Bluethmann, H. \& Alexander, J. In interleukin-4deficient mice, alum not only generates $\mathrm{T}$ helper 1 responses equivalent to Freund's complete adjuvant, but continues to induce T helper 2 cytokine production. Eur. J. Immunol. 26, 2062-2066 (1996).
46. MacLeod, M. K. et al. Memory CD4 T cells that express CXCR5 provide accelerated help to B cells. J. Immunol. 186, 2889-2896 (2011).

47. Serre, K. et al. Selective effects of NF-kB1 deficiency in $\mathrm{CD}^{+} \mathrm{T}$ cells on $\mathrm{T}_{\mathrm{H}} 2$ and $\mathrm{T}_{\mathrm{FH}}$ induction by alum-precipitated protein vaccines. Eur. J. Immunol. 41, 1573-1582 (2011)

48. Zhou, C. et al. Analysis of immunological mechanisms exerted by HBsAg-HBIG therapeutic vaccine combined with Adefovir in chronic hepatitis B patients. Hum. Vaccin. Immunother. 13, 1989-1996 (2017).

49. Hutchison, S. et al. Antigen depot is not required for alum adjuvanticity. FASEB J. 26, 1272-1279 (2012).

50. Moyer, T. J. et al. Engineered immunogen binding to alum adjuvant enhances humoral immunity. Nat. Med. 26, 430-440 (2020)

51. Marrack, P., McKee, A. S. \& Munks, M. W. Towards an understanding of the adjuvant action of aluminium. Nat. Rev. Immunol. 9, 287-293 (2009).

52. Gavin, A. L. et al. Adjuvant-enhanced antibody responses in the absence of Toll-like receptor signaling. Science 314, 1936-1938 (2006) This study demonstrates adjuvanticity in the absence of TLR stimulation.

53. Eisenbarth, S. C., Colegio, O. R., O'Connor, W. Sutterwala, F. S. \& Flavell, R. A. Crucial role for the Nalp3 inflammasome in the immunostimulatory properties of aluminium adjuvants. Nature $\mathbf{4 5 3}$, 1122-1126 (2008)

54. Li, H., Nookala, S. \& Re, F. Aluminum hydroxide adjuvants activate caspase- 1 and induce IL- $1 \beta$ and IL-18 release. J. Immunol. 178, 5271-5276 (2007).

55. McKee, A. S. et al. Alum induces innate immune responses through macrophage and mast cell sensors, but these sensors are not required for alum to act as an adjuvant for specific immunity. J. Immunol. 183. 4403-4414 (2009).

56. Kool, M. et al. Cutting edge: alum adjuvant stimulates inflammatory dendritic cells through activation of the NALP3 inflammasome. J. Immunol. 181, 3755-3759 (2008).

57. Franchi, L. \& Nunez, G. The Nlrp3 inflammasome is critical for aluminium hydroxide-mediated IL-1 secretion but dispensable for adjuvant activity. Eur. J. Immunol. 38, 2085-2089 (2008).

58. Schmitz, N., Kurrer, M. \& Kopf, M. The IL-1 receptor 1 is critical for $\mathrm{T}_{\mathrm{H}} 2$ cell type airway immune responses in a mild but not in a more severe asthma model. Eur. J. Immunol. 33, 991-1000 (2003)

59. Kool, M. et al. Alum adjuvant boosts adaptive immunity by inducing uric acid and activating inflammatory dendritic cells. J. Exp. Med. 205 869-882 (2008)

60. Munks, M. W. et al. Aluminum adjuvants elicit fibrin-dependent extracellular traps in vivo. Blood 116, 5191-5199 (2010).

61. Walls, R. S. Eosinophil response to alum adjuvants: involvement of T cells in non-antigen-dependent mechanisms. Proc. Soc. Exp. Biol. Med. 156, 431-435 (1977)

62. Marichal, T. et al. DNA released from dying host cells mediates aluminum adjuvant activity. Nat. Med. 17 996-1002 (2011).

This study is the first to show that critical cell death pathways are involved in the adjuvanticity of alum.

63. McKee, A. S. et al. Host DNA released in response to aluminum adjuvant enhances MHC class II-mediated antigen presentation and prolongs CD4 T-cell interactions with dendritic cells. Proc. Natl Acad. Sci. USA 110, E1122-E1131 (2013).

64. Noges, L. E., White, J., Cambier, J. C., Kappler, J. W. \& Marrack, P. Contamination of DNase preparations confounds analysis of the role of DNA in alum-adjuvanted vaccines. J. Immunol. 197, 1221-1230 (2016).

65. Xia, S. et al. Safety and immunogenicity of an inactivated SARS-CoV-2 vaccine, BBIBP-CorV: a randomised, double-blind, placebo-controlled, phase 1/2 trial. Lancet Infect. Dis. 21, 39-51 (2021).

66. Zhang, Y. et al. Safety, tolerability, and immunogenicity of an inactivated SARS-CoV-2 vaccine in healthy adults aged 18-59 years: a randomised, double-blind, placebo-controlled, phase $1 / 2$ clinical trial Lancet Infect. Dis. 21, 181-192 (2020).

67. HogenEsch, H., O'Hagan, D. T. ¿ Fox, C. B. Optimizing the utilization of aluminum adjuvants in vaccines: you might just get what you want. NPJ Vaccines 3, 51 (2018)

68. O'Hagan, D. T., Ott, G. S., Nest, G. V., Rappuoli, R. $\&$ Giudice, G. D. The history of MF59 ${ }^{\circledR}$ adjuvant: a phoenix that arose from the ashes. Expert Rev. Vaccines 12, 13-30 (2013).
69. O'Hagan, D. T., Ott, G. S., De Gregorio, E. \& Seubert, A. The mechanism of action of MF59 an innately attractive adjuvant formulation. Vaccine 30, 4341-4348 (2012).

70. Calabro, S. et al. Vaccine adjuvants alum and MF59 induce rapid recruitment of neutrophils and monocytes that participate in antigen transport to draining lymph nodes. Vaccine 29, 1812-1823 (2011).

71. Mosca, F. et al. Molecular and cellular signatures of human vaccine adjuvants. Proc. Natl Acad. Sci. USA 105, 10501-10506 (2008).

72. Liang, F. et al. Vaccine priming is restricted to draining lymph nodes and controlled by adjuvant-mediated antigen uptake. Sci. Transl Med. 9, eaal2094 (2017)

73. Vono, M. et al. The adjuvant MF59 induces ATP release from muscle that potentiates response to vaccination. Proc. Natl Acad. Sci. USA 110 21095-21100 (2013).

74. Seubert, A. et al. Adjuvanticity of the oil-in-water emulsion MF59 is independent of NIrp3 inflammasome but requires the adaptor protein MyD88. Proc. Natl Acad. Sci. USA 108, 11169-11174 (2011).

75. Ellebedy, A. H. et al. Inflammasome-independent role of the apoptosis-associated speck-like protein containing CARD (ASC) in the adjuvant effect of MF59. Proc. Natl Acad. Sci. USA 108, 2927-2932 (2011).

76. Kim, E. H. et al. Squalene-based adjuvants stimulate CD8 T cell, but not antibody responses, through a RIPK3-dependent pathway. eLife 9, e52687 (2020). This study demonstrates the role of RIPK3 in mediating $\mathrm{CD8}^{+} \mathrm{T}$ cell responses induced by MF59, a squalene-based adjuvant.

77. Didierlaurent, A. M. et al. AS04, an aluminum saltand TLR4 agonist-based adjuvant system, induces a transient localized innate immune response leading to enhanced adaptive immunity. J. Immunol. 183. 6186-6197 (2009).

78. Garcon, N. et al. Development of an AS04-adjuvanted HPV vaccine with the adjuvant system approach. BioDrugs 25, 217-226 (2011).

79. Kundi, M. New hepatitis B vaccine formulated with an improved adjuvant system. Expert Rev. Vaccines 6 , 133-140 (2007).

80. Skinner, S. R. et al. Human papillomavirus (HPV) 16/18 AS04-adjuvanted vaccine for the prevention of cervical cancer and HPV-related diseases. Expert Rev. Vaccines 15, 367-387 (2016).

81. Tong, N. K. et al. Immunogenicity and safety of an adjuvanted hepatitis B vaccine in prehemodialysis and hemodialysis patients. Kidney Int 68, 2298-2303 (2005).

82. Naud, P. S. et al. Sustained efficacy, immunogenicity, and safety of the HPV-16/18 AS04-adjuvanted vaccine: final analysis of a long-term follow-up study up to 9.4 years post-vaccination. Hum. Vaccin. Immunother. 10, 2147-2162 (2014).

83. Ryser, M. et al. Post-hoc analysis from phase III trials of human papillomavirus vaccines: considerations on impact on non-vaccine types. Expert Rev. Vaccines 18 309-322 (2019).

84. Garcon, N. \& Di Pasquale, A. From discovery to licensure, the adjuvant system story. Hum. Vaccin. Immunother. 13, 19-33 (2017).

85. Sanofi. Sanofi and GSK initiate Phase $1 / 2$ clinical trial of COVID-19 adjuvanted recombinant protein-based vaccine candidate. Sanofi https://www.sanofi.com/ en/media-room/press-releases/2020/2020-09-0307-00-00 (2020)

86. Ward, B. J. et al. Phase 1 trial of a candidate recombinant virus-like particle vaccine for covid-19 disease produced in plants. Preprint at medRxiv https://doi.org/10.1101/2020.11.04.20226282 (2020)

87. Garcon, N., Vaughn, D. W. \& Didierlaurent, A. M. Development and evaluation of AS03, an adjuvant system containing $\alpha$-tocopherol and squalene in an oil-in-water emulsion. Expert Rev. Vaccines 11 349-366 (2012)

88. Morel, S. et al. Adjuvant System ASO3 containing a-tocopherol modulates innate immune response and leads to improved adaptive immunity. Vaccine 29, 2461-2473 (2011)

89. Orr, M. T. \& Fox, C. B. ASO3 stresses out macrophages: commentary on 'Activation of the endoplasmic reticulum stress sensor IRE $1 \alpha$ by the vaccine adjuvant ASO3 contributes to its immunostimulatory properties'. NPJ Vaccines 3, 27 (2018).

90. Howard, L. M. et al. Cell-based systems biology analysis of human AS03-adjuvanted $\mathrm{H} 5 \mathrm{~N} 1$ avian influenza vaccine responses: a phase I randomized controlled trial. PLOS ONE 12 $0167488(2017)$ 
91. Sobolev, O. et al. Adjuvanted influenza-H1N1 vaccination reveals lymphoid signatures of age-dependent early responses and of clinical adverse events. Nat. Immunol. 17, 204-213 (2016).

92. Givord, C. et al. Activation of the endoplasmic reticulum stress sensor IRE 1 a by the vaccine adjuvant $\mathrm{ASO} 3$ contributes to its immunostimulatory properties. NPJ Vaccines 3, 20 (2018).

93. Lal, H. et al. Efficacy of an adjuvanted herpes zoster subunit vaccine in older adults. N. Engl. J. Med. 372, 2087-2096 (2015)

94. Olotu, A. et al. Seven-year efficacy of RTS,S/AS01 malaria vaccine among young African children. N. Engl. J. Med. 374, 2519-2529 (2016).

95. Kensil, C. R., Patel, U., Lennick, M. \& Marciani, D. Separation and characterization of saponins with adjuvant activity from Quillaja saponaria Molina cortex. J. Immunol. 146, 431-437 (1991).

96. Newman, M. J. et al. Saponin adjuvant induction of ovalbumin-specific CD8 ${ }^{+}$cytotoxic T lymphocyte responses. J. Immunol 148, 2357-2362 (1992)

97. Wu, J. Y. et al. Saponin adjuvant enhancement of antigen-specific immune responses to an experimental HIV-1 vaccine. J. Immunol. 148 1519-1525 (1992).

98. Detienne, S. et al. Central role of $\mathrm{CD}_{169}{ }^{+}$lymph node resident macrophages in the adjuvanticity of the QS-21 component of AS01. Sci. Rep. 6, 39475 (2016).

99. Marty-Roix, R. et al. Identification of QS-21 as an inflammasome-activating molecular component of saponin adjuvants. J. Biol. Chem. 291, 1123-1136 (2016).

100. Welsby, I. et al. Lysosome-dependent activation of human dendritic cells by the vaccine adjuvant OS- 21 . Front. Immunol. 7, 663 (2016).

101. Coccia, M. et al. Cellular and molecular synergy in AS01-adjuvanted vaccines results in an early IFN $\gamma$ response promoting vaccine immunogenicity. NPJ Vaccines 2, 25 (2017).

102. O'Hagan, D. T., Friedland, L. R., Hanon, E. \& Didierlaurent, A. M. Towards an evidence based approach for the development of adjuvanted vaccines. Curr. Opin. Immunol. 47, 93-102 (2017).

103. Guiducci, C. et al. Properties regulating the nature of the plasmacytoid dendritic cell response to Toll-like receptor 9 activation. J. Exp. Med. 203, 1999-2008 (2006).

104. No authors listed]. A two-dose hepatitis $B$ vaccine for adults (Heplisav-B). JAMA 319, 822-823 (2018)

105. Barry, M. \& Cooper, C. Review of hepatitis B surface antigen-1018 ISS adjuvant-containing vaccine safety and efficacy. Expert Opin. Biol. Ther. 7, 1731-1737 (2007).

106. Campbell, J. D. et al. CpG-containing immunostimulatory DNA sequences elicit TNF-a-dependent toxicity in rodents but not in humans. J. Clin. Invest. 119, 2564-2576 (2009).

107. Hartmann, G. Nucleic acid immunity. Adv. Immunol. 133, 121-169 (2017).

108. Hou, B., Reizis, B. \& DeFranco, A. L. Toll-like receptors activate innate and adaptive immunity by using dendritic cell-intrinsic and -extrinsic mechanisms. Immunity 29, 272-282 (2008)

109. Akondy, R. S. et al. Initial viral load determines the magnitude of the human CD8 T cell response to yellow fever vaccination. Proc. Natl Acad. Sci. USA 112, 3050-3055 (2015). This study delineates the role of the magnitude of the antigen in shaping CD8 $\mathrm{T}$ cell immunity.

110. Miller, J. D. et al. Human effector and memory CD8 $\mathrm{T}$ cell responses to smallpox and yellow fever vaccines. Immunity 28, 710-722 (2008)

111. Datta, S. K. et al. A subset of Toll-like receptor ligands induces cross-presentation by bone marrow-derived dendritic cells. J. Immunol. 170 4102-4110 (2003).

112. Jelinek, I. et al. TLR3-specific double-stranded RNA oligonucleotide adjuvants induce dendritic cell cross-presentation, CTL responses, and antiviral protection. J. Immunol. 186, 2422-2429 (2011).

113. Oh, J. Z., Kurche, J. S., Burchill, M. A. \& Kedl, R. M. TLR7 enables cross-presentation by multiple dendritic cell subsets through a type I IFN-dependent pathway. Blood 118, 3028-3038 (2011).

114. Bonifaz, L. C. et al. In vivo targeting of antigens to maturing dendritic cells via the DEC-205 receptor improves T cell vaccination. J. Exp. Med. 199, 815-824 (2004).
115. Idoyaga, J. et al. Comparable $T$ helper $1\left(T_{H} 1\right)$ and CD8 T-cell immunity by targeting HIV gag p 24 to CD8 dendritic cells within antibodies to Langerin, DEC205, and Clec9A. Proc. Natl Acad. Sci. USA 108, 2384-2389 (2011).

116. Kwissa, M. et al. Adjuvanting a DNA vaccine with a TLR9 ligand plus FIt3 ligand results in enhanced cellular immunity against the simian immunodeficiency virus. J. Exp. Med. 204, 2733-2746 (2007).

117. Wille-Reece, U. et al. HIV Gag protein conjugated to a Toll-like receptor $7 / 8$ agonist improves the magnitude and quality of $\mathrm{T}_{H} 1$ and $C D 8^{+} \mathrm{T}$ cell responses in nonhuman primates. Proc. Natl Acad. Sci. USA 102, 15190-15194 (2005).

This study is one of the first to demonstrate the adjuvant activity of a TLR ligand in NHPs.

118. Wille-Reece, U., Wu, C. Y., Flynn, B. J., Kedl, R. M. \& Seder, R. A. Immunization with HIV-1 Gag protein conjugated to a TLR7/8 agonist results in the generation of HIV-1 Gag-specific $\mathrm{T}_{\mathrm{H}} 1$ and $\mathrm{CD} 8^{+} \mathrm{T}$ cell responses. J. Immunol. 174, 7676-7683 (2005).

119. Couch, R. B. et al. Superior antigen-specific CD4 ${ }^{+}$T-cell response with AS03-adjuvantation of a trivalent influenza vaccine in a randomised trial of adults aged 65 and older. BMC Infect. Dis. 14, 425 (2014).

120. Leroux-Roels, G. et al. Impact of adjuvants on CD4 $T$ cell and $B$ cell responses to a protein antigen vaccine: results from a phase II, randomized, multicenter trial. Clin. Immunol. 169, 16-27 (2016).

121. Boyer, J. D. et al. In vivo protective anti-HIV immune responses in non-human primates through DNA immunization. J. Med. Primatol. 25, 242-250 (1996).

122. Lu, S. et al. Simian immunodeficiency virus DNA vaccine trial in macaques. J. Virol. 70, 3978-3991 (1996).

123. MacGregor, R. R. et al. First human trial of a DNA-based vaccine for treatment of human immunodeficiency virus type 1 infection: safety and host response. J. Infect. Dis. 178, 92-100 (1998).

124. Parks, C. L., Picker, L. J. \& King, C. R. Development of replication-competent viral vectors for HIV vaccine delivery. Curr. Opin. HIV AIDS 8, 402-411 (2013).

125. Barouch, D. H. et al. Evaluation of a mosaic HIV-1 vaccine in a multicentre, randomised, double-blind, placebo-controlled, phase $1 / 2$ a clinical trial (APPROACH) and in rhesus monkeys (NHP 13-19). Lancet 392, 232-243 (2018).

126. Sahin, U. et al. COVID-19 vaccine BNT162b1 elicits human antibody and $\mathrm{T}_{\mathrm{H}} 1 \mathrm{~T}$-cell responses. Nature 586, 594-599 (2020).

This phase I/II clinical trial of the Pfizer/BioNTech COVID-19 mRNA vaccine demonstrates potent neutralizing antibody titres and a relatively high magnitude of antigen-specific $\mathrm{CD8}^{+} \mathrm{T}$ cell response.

127. Carbone, F. R. Tissue-resident memory T cells and fixed immune surveillance in nonlymphoid organs. J. Immunol. 195, 17-22 (2015).

128. Masopust, D. \& Soerens, A. G. Tissue-resident T cells and other resident leukocytes. Annu. Rev. Immunol. 37, 521-546 (2019)

129. Arunachalam, P. S. et al. T cell-inducing vaccine durably prevents mucosal SHIV infection even with lower neutralizing antibody titers. Nat. Med. 26, 932-940 (2020).

This study demonstrates that vaccines that stimulate a high magnitude of antigen-specific $\mathrm{CD}^{+} \mathrm{T}_{\mathrm{RM}}$ cell response lower the threshold of neutralizing antibody response required for protection, suggesting a potential synergy between $T_{R M}$ cells and antibody responses.

130. Ho, N. I., Huis In 't Veld, L. G. M., Raaijmakers, T. K. $\delta$ Adema, G. J. Adjuvants enhancing cross-presentation by dendritic cells: the key to more effective vaccines? Front. Immunol. 9, 2874 (2018).

131. Klechevsky, E. \& Banchereau, J. Human dendritic cells subsets as targets and vectors for therapy. Ann. NY Acad. Sci. 1284, 24-30 (2013).

132. Seubert, A., Monaci, E., Pizza, M., O'Hagan, D. T. \& Wack, A. The adjuvants aluminum hydroxide and MF59 induce monocyte and granulocyte chemoattractants and enhance monocyte differentiation toward dendritic cells. J. Immunol. 180, 5402-5412 (2008).

133. Tian, M. et al. B cell-intrinsic MyD88 signaling promotes initial cell proliferation and differentiation to enhance the germinal center response to a virus-like particle. J. Immunol. 200, 937-948 (2018).
134. Cantisani, R. et al. Vaccine adjuvant MF59 promotes retention of unprocessed antigen in lymph node macrophage compartments and follicular dendritic cells. J. Immunol. 194, 1717-1725 (2015).

135. Neutra, M. R., Frey, A. \& Kraehenbuhl, J. P. Epithelial M cells: gateways for mucosal infection and immunization. Cell 86, 345-348 (1996).

136. Fritz, J. H., Ferrero, R. L., Philpott, D. J. \& Girardin, S. E. Nod-like proteins in immunity, inflammation and disease. Nat. Immunol. 7. 1250-1257 (2006).

137. Yong, H. Y. \& Luo, D. RIG-I-like receptors as novel targets for pan-antivirals and vaccine adjuvants against emerging and re-emerging viral infections. Front. Immunol. 9, 1379 (2018).

138. Chiffoleau, E. C-type lectin-like receptors as emerging orchestrators of sterile inflammation represent potential therapeutic targets. Front. Immunol. 9, 227 (2018).

139. Dubensky, T. W. Jr. Kanne, D. B. \& Leong, M. L. Rationale, progress and development of vaccines utilizing STING-activating cyclic dinucleotide adjuvants. Ther. Adv. Vaccines 1, 131-143 (2013).

140. Maisonneuve, C., Bertholet, S., Philpott, D. J. \& De Gregorio, E. Unleashing the potential of NOD- and Toll-like agonists as vaccine adjuvants. Proc. Natl Acad. Sci. USA 111, 12294-12299 (2014).

141. Munoz-Planillo, R. et al. $\mathrm{K}^{+}$efflux is the common trigger of NLRP3 inflammasome activation by bacterial toxins and particulate matter. Immunity 38 1142-1153 (2013).

142. Gutjahr, A. et al. The STING ligand cGAMP potentiates the efficacy of vaccine-induced $\mathrm{CD}^{+} \mathrm{T}$ cells. JCl Insight 4, e125107 (2019)

143. Kandasamy, M. et al. RIG-I signaling is critical for efficient polyfunctional $\mathrm{T}$ cell responses during influenza virus infection. PLoS Pathog. 12, e 1005754 (2016).

144. Suthar, M. S. et al. The RIG-I-like receptor LGP2 controls $\mathrm{CD} 8{ }^{+} \mathrm{T}$ cell survival and fitness. Immunity 37 . 235-248 (2012).

145. Chen, Y. G. et al. $N^{6}$-Methyladenosine modification controls circular RNA immunity. Mol. Cell 76, 96-109.e9 (2019).

146. De Lorenzo, G., Ferrari, S., Cervone, F. \& Okun, E. Extracellular DAMPs in plants and mammals: immunity, tissue damage and repair. Trends Immunol. 39, 937-950 (2018)

147. Gallo, P. M. \& Gallucci, S. The dendritic cell response to classic, emerging, and homeostatic danger signals. Implications for autoimmunity. Front. Immunol. 4, 138 (2013).

148. Bevan, M. J. Cross-priming for a secondary cytotoxic response to minor $\mathrm{H}$ antigens with $\mathrm{H}-2$ congenic cells which do not cross-react in the cytotoxic assay. J. Exp. Med. 143, 1283-1288 (1976).

149. Albert, M. L., Sauter, B. \& Bhardwaj, N. Dendritic cells acquire antigen from apoptotic cells and induce class I-restricted CTLs. Nature 392, 86-89 (1998).

150. Tang, D., Kang, R., Berghe, T. V., Vandenabeele, P. \& Kroemer, $\mathrm{G}$. The molecular machinery of regulated cell death. Cell Res. 29, 347-364 (2019).

151. Cho, Y. S. et al. Phosphorylation-driven assembly of the RIP1-RIP3 complex regulates programmed necrosis and virus-induced inflammation. Cell 137 1112-1123 (2009).

152. He, S. et al. Receptor interacting protein kinase-3 determines cellular necrotic response to TNF-a. Cell 137, 1100-1111 (2009)

153. Zhang, D. W. et al. RIP3, an energy metabolism regulator that switches TNF-induced cell death from apoptosis to necrosis. Science 325, 332-336 (2009).

154. Yatim, N. et al. RIPK1 and NF-кB signaling in dying cells determines cross-priming of $\mathrm{CD} 8^{+} \mathrm{T}$ cells. Science 350, 328-334 (2015).

155. Pearce, E. J. \& Everts, B. Dendritic cell metabolism Nat. Rev. Immunol. 15, 18-29 (2015).

156. Sinclair, C. et al. mTOR regulates metabolic adaptation of APCs in the lung and controls the outcome of allergic inflammation. Science 357, 1014-102 (2017).

157. Cao, W. et al. Toll-like receptor-mediated induction of type I interferon in plasmacytoid dendritic cells requires the rapamycin-sensitive PI(3)K-mTOR-p70S6K pathway. Nat. Immunol. 9, 1157-1164 (2008).

158. Ravindran, R. et al. Vaccine activation of the nutrient sensor GCN2 in dendritic cells enhances antigen presentation. Science 343, 313-317 (2014). This study provides insights into role of GCN2, a predictor of YF-17D immunogenicity in humans. 
159. Ravindran, R. et al. The amino acid sensor GCN2 controls gut inflammation by inhibiting inflammasome activation. Nature 531, 523-527 (2016).

160. Halaby, M. J. et al. GCN2 drives macrophage and MDSC function and immunosuppression in the tumor microenvironment. Sci. Immunol. 4, eaax8189 (2019)

161. Netea, M. G. et al. Trained immunity: a program of innate immune memory in health and disease. Science 352, aaf1098 (2016)

162. Netea, M. G., Latz, E., Mills, K. H. \& O’Neill, L. A Innate immune memory: a paradigm shift in understanding host defense. Nat. Immunol. 16, 675-679 (2015)

163. Netea, M. G. \& van der Meer, J. W. Trained immunity: an ancient way of remembering. Cell Host Microbe 21 297-300 (2017).

164. Cerwenka, A. \& Lanier, L. L. Natural killer cell memory in infection, inflammation and cancer. Nat. Rev. Immunol. 16, 112-123 (2016).

165. Kleinnijenhuis, J. et al. Bacille Calmette-Guérin induces NOD2-dependent nonspecific protection from reinfection via epigenetic reprogramming of monocytes. Proc. Natl Acad. Sci. USA 109, 17537-17542 (2012).

This study is the first to demonstrate trained immunity in monocytes of BCG-vaccinated humans.

166. Arts, R. J. W. et al. BCG vaccination protects against experimental viral infection in humans through the induction of cytokines associated with trained immunity. Cell Host Microbe 23, 89-100.e5 (2018).

167. Mulder, W. J. M., Ochando, J., Joosten, L. A. B. Fayad, Z. A. \& Netea, M. G. Therapeutic targeting of trained immunity, Nat. Rev. Drug Discov. 18 553-566 (2019)

168. Crotty, S. T follicular helper cell biology: a decade of discovery and diseases. Immunity 50, 1132-1148 (2019).

169. Mesin, L., Ersching, J. \& Victora, G. D. Germinal center B cell dynamics. Immunity 45, 471-482 (2016)

170. Vinuesa, C. G., Linterman, M. A., Yu, D. \& MacLennan, I. C. Follicular helper T cells. Annu. Rev. Immunol. 34, 335-368 (2016)

171. Bernasconi, N. L., Traggiai, E. \& Lanzavecchia, A. Maintenance of serological memory by polyclonal activation of human memory B cells. Science $\mathbf{2 9 8}$ 2199-2202 (2002)

172. Das, A. et al. Follicular dendritic cell activation by TLR ligands promotes autoreactive $B$ cell responses. Immunity 46, 106-119 (2017)

173. Garin, A. et al. Toll-like receptor 4 signaling by follicular dendritic cells is pivotal for germinal center onset and affinity maturation. Immunity 33, 84-95 (2010).

174. June, C. H., Bluestone, J. A., Nadler, L. M. \& Thompson, C. B. The B7 and CD28 receptor families. Immunol. Today 15, 321-331 (1994).

175. Mueller, D. L., Jenkins, M. K. \& Schwartz, R. H. Clonal expansion versus functional clonal inactivation: a costimulatory signalling pathway determines the outcome of T cell antigen receptor occupancy. Annu. Rev. Immunol. 7, 445-480 (1989).

176. Talmage, D. W., Dart, G., Radovich, J. \& Lafferty, K. J. Activation of transplant immunity: effect of donor leukocytes on thyroid allograft rejection. Science 191 385-388 (1976)

177. Monto, A. S., Malosh, R. E., Petrie, J. G. \& Martin, E. T. The doctrine of original antigenic sin: separating good from evil. J. Infect. Dis. 215, 1782-1788 (2017).

178. Henry, C., Palm, A. E., Krammer, F. \& Wilson, P. C. From original antigenic sin to the universal influenza virus vaccine. Trends Immunol. 39, 70-79 (2018).

179. Krammer, F. \& Palese, P. Advances in the development of influenza virus vaccines. Nat. Rev. Drug Discov. 14 167-182 (2015).

180. Ellebedy, A. H. et al. Adjuvanted H5N1 influenza vaccine enhances both cross-reactive memory $B$ cell and strain-specific naive $B$ cell responses in humans. Proc. Natl Acad. Sci. USA 117, 17957-17964 (2020).

181. de Jong, S. E., Olin, A. \& Pulendran, B. The impact of the microbiome on immunity to vaccination in humans. Cell Host Microbe 28, 169-179 (2020).

182. Pulendran, B. Systems vaccinology: probing humanity's diverse immune systems with vaccines. Proc. Natl Acad. Sci. USA 111, 12300-12306 (2014).

183. Pulendran, B. \& Davis, M. M. The science and medicine of human immunology. Science 369 , eaay4014 (2020)
184. Sasaki, S. et al. Limited efficacy of inactivated influenza vaccine in elderly individuals is associated with decreased production of vaccine-specific antibodies. J. Clin. Invest. 121, 3109-3119 (2011).

185. Seidman, J. C., Richard, S. A., Viboud, C. \& Miller, M. A. Quantitative review of antibody response to inactivated seasonal influenza vaccines. Influenza Other Respir. Viruses 6, 52-62 (2012).

186. Amanna, I. J. Balancing the efficacy and safety of vaccines in the elderly. Open Longev. Sci. 6, 64-72 (2012).

187. Weinberger, B. Adjuvant strategies to improve vaccination of the elderly population. Curr. Opin. Pharmacol. 41, 34-41 (2018).

188. Heyward, W. L. et al. Immunogenicity and safety of an investigational hepatitis B vaccine with a Toll-like receptor 9 agonist adjuvant (HBsAg-1018) compared to a licensed hepatitis $B$ vaccine in healthy adults 40-70 years of age. Vaccine 31, 5300-5305 (2013).

189. Dowling, D. J. et al. TLR7/8 adjuvant overcomes newborn hyporesponsiveness to pneumococcal conjugate vaccine at birth. JCI Insight 2, e91020 (2017).

190. Levy, O. Innate immunity of the newborn: basic mechanisms and clinical correlates. Nat. Rev. Immunol. 7, 379-390 (2007)

191. Sanchez-Schmitz, G. \& Levy, O. Development of newborn and infant vaccines. Sci. Transl Med. 3, 90ps27 (2011)

192. Belkaid, Y. \& Hand, T. W. Role of the microbiota in immunity and inflammation. Cell 157, 121-141 (2014).

193. Collins, N. \& Belkaid, Y. Do the microbiota influence vaccines and protective immunity to pathogens? Engaging our endogenous adjuvants. Cold Spring Harb. Perspect. Biol. 10, a028860 (2018)

194. Hagan, T. et al. Antibiotics-driven gut microbiome perturbation alters immunity to vaccines in humans. Cell 178, 1313-1328.e13 (2019).

This study is the first to reveal the role of microbiota in the response to influenza vaccination in humans.

195. Vitetta, L., Saltzman, E. T., Thomsen, M., Nikov, T $\&$ Hall, S. Adjuvant probiotics and the intestinal microbiome: enhancing vaccines and immunotherapy outcomes. Vaccines 5, 50 (2017)

196. Ericsson, D. J. et al. Identification of small peptides mimicking the R2 C-terminus of Mycobacterium tuberculosis ribonucleotide reductase. J. Pept. Sci. 16, 159-164 (2010).

197. Guan, Y., Omueti-Ayoade, K., Mutha, S. K. Hergenrother, P. J. \& Tapping, R. I. Identification of novel synthetic Toll-like receptor 2 agonists by high throughput screening. J. Biol. Chem. 285 23755-23762 (2010).

198. Wu, T. Y. Strategies for designing synthetic immune agonists. Immunology 148, 315-325 (2016).

199. Wu, T. Y. et al. Rational design of small molecules as vaccine adjuvants. Sci. Transl Med. 6, 263ra160 (2014).

200. Brito, L. A. \& O'Hagan, D. T. Designing and building the next generation of improved vaccine adjuvants. J. Control. Rel. 190, 563-579 (2014).

201. Ramanjulu, J. M. et al. Design of amidobenzimidazole STING receptor agonists with systemic activity. Nature 564, 439-443 (2018)

202. Salyer, A. C. et al. Identification of adjuvantic activity of amphotericin B in a novel, multiplexed poly-TLR/NLR high-throughput screen. PLOS ONE 11, e0149848 (2016).

203. Lodaya, R. N. et al. Stable nanoemulsions for the delivery of small molecule immune potentiators. J. Pharm. Sci. 107, 2310-2314 (2018)

204. Irvine, D. J., Aung, A. \& Silva, M. Controlling timing and location in vaccines. Adv. Drug Deliv. Rev. 158 91-115 (2020).

205. Irvine, D. J. \& Read, B. J. Shaping humoral immunity to vaccines through antigen-displaying nanoparticles. Curr. Opin. Immunol. 65, 1-6 (2020).

206. Tokatlian, T. et al. Innate immune recognition of glycans targets HIV nanoparticle immunogens to germinal centers. Science 363, 649-654 (2019).

207. Roth, G. A. et al. Injectable hydrogels for sustained codelivery of subunit vaccines enhance humoral immunity. ACS Cent. Sci. 6, 1800-1812 (2020).

208. Van Hoeven, N. et al. A formulated TLR7/8 agonist is a flexible, highly potent and effective adjuvant for pandemic influenza vaccines. Sci. Rep. 7, 46426 (2017).
209. Fox, C. B. et al. Adsorption of a synthetic TLR7/8 ligand to aluminum oxyhydroxide for enhanced vaccine adjuvant activity: a formulation approach J. Control. Rel. 244, 98-107 (2016).

210. Jain, S., O'Hagan, D. T. \& Singh, M. The long-term potential of biodegradable poly(lactide-co-glycolide) microparticles as the next-generation vaccine adjuvant. Expert Rev. Vaccines 10, 1731-1742 (2011)

211. Cortez, A. et al. Incorporation of phosphonate into benzonaphthyridine Toll-like receptor 7 agonists for adsorption to aluminum hydroxide. J. Med. Chem. 59, 5868-5878 (2016).

212. Gonzalez-Lopez, A. et al. Adjuvant effect of TLR7 agonist adsorbed on aluminum hydroxide (AS37): a phase I randomized, dose escalation study of an AS37-adjuvanted meningococcal C conjugated vaccine. Clin. Immunol. 209, 108275 (2019).

213. Didierlaurent, A. M. et al. Adjuvant system AS01: helping to overcome the challenges of modern vaccines. Expert Rev. Vaccines 16, 55-63 (2017).

214. Davis, M. M., Tato, C. M. \& Furman, D. Systems immunology: just getting started. Nat. Immunol. 18, 725-732 (2017)

215. Gaucher, D. et al. Yellow fever vaccine induces integrated multilineage and polyfunctional immune responses. J. Exp. Med. 205, 3119-3131 (2008).

216. Nakaya, H. I. et al. Systems biology of vaccination for seasonal influenza in humans. Nat. Immunol. 12 , 786-795 (2011).

217. Nakaya, H. I. et al. Systems analysis of immunity to influenza vaccination across multiple years and in diverse populations reveals shared molecular signatures. Immunity 43, 1186-1198 (2015).

218. Tsang, J. S. et al. Global analyses of human immune variation reveal baseline predictors of postvaccination responses. Cell 157, 499-513 (2014). This paper shows the potential of the systems biology approach in determining baseline predictors of vaccine responses.

219. Kotliarov, Y et al. Broad immune activation underlies shared set point signatures for vaccine responsiveness in healthy individuals and disease activity in patients with lupus. Nat. Med. 26, 618-629 (2020).

220. Franco, L. M. et al. Integrative genomic analysis of the human immune response to influenza vaccination. Life 2, e00299 (2013).

221. Furman, D. et al. Apoptosis and other immune biomarkers predict influenza vaccine responsiveness. Mol. Syst. Biol. 9, 659 (2013).

222. Vahey, M. T. et al. Expression of genes associated with immunoproteasome processing of major histocompatibility complex peptides is indicative of protection with adjuvanted RTS,S malaria vaccine. J. Infect. Dis. 201, 580-589 (2010).

223. Kazmin, D. et al. Systems analysis of protective immune responses to RTS,S malaria vaccination in humans. Proc. Natl Acad. Sci. USA 114, 2425-2430 (2017).

224. van den Berg, R. A. et al. Predicting RTS,S vaccine-mediated protection from transcriptomes in a malaria-challenge clinical trial. Front. Immunol. 8, 557 (2017).

225. Li, S. et al. Molecular signatures of antibody responses derived from a systems biology study of five human vaccines. Nat. Immunol. 15, 195-204 (2014).

226. Obermoser, G. et al. Systems scale interactive exploration reveals quantitative and qualitative differences in response to influenza and pneumococcal vaccines. Immunity 38, 831-844 (2013).

227. Li, S. et al. Metabolic phenotypes of response to vaccination in humans. Cell 169, 862-877.e17 (2017).

228. Oh, J. Z. et al. TLR5-mediated sensing of gut microbiota is necessary for antibody responses to seasonal influenza vaccination. Immunity 41 478-492 (2014).

229. Caskey, M. et al. Synthetic double-stranded RNA induces innate immune responses similar to a live viral vaccine in humans. J. Exp. Med. 208, 2357-2366 (2011).

This small-scale human study of a novel adjuvant candidate highlights the value of proposed phase $0 / I$ human studies using systems approaches.

230. Keech, C. et al. Phase 1-2 trial of a SARS-CoV-2 recombinant spike protein nanoparticle vaccine. N. Engl. J. Med. 383, 2320-2332 (2020).

231. Garcia, A. \& Lema, D. An updated review of ISCOMSTM and ISCOMATRIXTM vaccines. Curr. Pharm. Des. 22, 6294-6299 (2016). 
232. Wagar, L. E. et al. Modeling human adaptive immune responses with tonsil organoids. Nat. Med. 27, 125-135 (2021)

233. Ella, R. et al. Safety and immunogenicity of an inactivated SARS-CoV-2 vaccine, BBV152: a doubleblind, randomised, phase 1 trial. Lancet Infect Dis. https://doi.org/10.1016/S1473-3099(20)30942-7 (2021).

234. Ramon, G. On diphtheria toxin and toxoid [French]. Ann. Inst. Pasteur 38, 13 (1924).

235. Pasquale, A. D., Preiss, S., Silva, F. T. D. \& Garçon, N. Vaccine adjuvants: from 1920 to 2015 and beyond. Vaccines 3, 320-343 (2015)

236. Glenny, A. T., Pope, C. G., Waddington, H. \& Wallace, U. Immunological notes. XVII-XXIV. J. Pathol. Bacteriol. 29, 31-40 (1926)

237. Rao, V. R. et al. Clade C HIV-1 isolates circulating in Southern Africa exhibit a greater frequency of dicysteine motif-containing Tat variants than those in Southeast Asia and cause increased neurovirulence. Retrovirology 10, 61 (2013).

238. Nakaya, H. I. et al. Systems biology of immunity to MF59-adjuvanted versus nonadjuvanted trivalent seasonal influenza vaccines in early childhood. Proc Natl Acad Sci. USA 113, 1853-1858 (2016).

239. Nanishi, E., Dowling, D. J. \& Levy, O. Toward precision adjuvants: optimizing science and safety. Curr. Opin. Pediatr. 32, 125-138 (2020).

\section{Acknowledgements}

B.P. acknowledges support from the National Institutes of Health $(\mathrm{NIH})$ and the Bill and Melinda Gates Foundation for some of the work described in this Review.

\section{Author contributions}

B.P. conceived and wrote the first draft of the article. B.P. and P.S.A. researched data for the article. B.P., P.S.A. and D.T.O provided substantial contribution to discussion of content and wrote the article. All authors edited and reviewed the article before submission.

Competing interests

D.T.O is a paid employee of CSK. B.P. serves on the External Immunology Network of CSK and is on the scientific advisory board of Medicago.

Peer review information

Nature Reviews Drug Discovery thanks Amy S. McKee and the other, anonymous, reviewers for their contribution to the peer review of this work.

Publisher's note

Springer Nature remains neutral with regard to jurisdictional claims in published maps and institutional affiliations.

(C) Springer Nature Limited 2021 